\title{
Jaccard and Dice Similarity Measures Based on Novel Complex Dual Hesitant Fuzzy Sets and Their Applications
}

\author{
Tahir Mahmood, ${ }^{1}$ Ubaid Ur Rehman, ${ }^{1}$ Zeeshan Ali, ${ }^{1}$ and Ronnason Chinram $\mathbb{D}^{2,3}$ \\ ${ }^{1}$ Department of Mathematics and Statistics, International Islamic University, Islamabad, Pakistan \\ ${ }^{2}$ Algebra and Applications Research Unit, Division of Computational Science, Faculty of Science, Prince of Songkla University, \\ Hat Yai, Songkhla 90110, Thailand \\ ${ }^{3}$ Centre of Excellence in Mathematics, Si Ayuthaya Road, Bangkok 10400, Thailand
}

Correspondence should be addressed to Ronnason Chinram; ronnason.c@psu.ac.th

Received 16 August 2020; Revised 12 September 2020; Accepted 9 October 2020; Published 11 November 2020

Academic Editor: Chonghui Zhang

Copyright (c) 2020 Tahir Mahmood et al. This is an open access article distributed under the Creative Commons Attribution License, which permits unrestricted use, distribution, and reproduction in any medium, provided the original work is properly cited.

\begin{abstract}
Complex dual hesitant fuzzy set (CDHFS) is a combination of two modifications, called complex fuzzy set (CFS) and dual hesitant fuzzy set (DHFS). CDHFS makes two degrees, called membership valued and nonmembership valued in the form of a finite subset of a unit disc in the complex plane, and is a capable method to solve uncertain and unpredictable information in real-life problems. The goal of this study is to describe the notion of CDHFS and its operational laws. The novel approach of the complex intervalvalued dual hesitant fuzzy set (CIvDHFS) and its fundamental laws are also described and defended with the help of an example. Further, the vector similarity measures (VSMs), weighted vector similarity measures (WVSMs), hybrid vector similarity measure, and weighted hybrid vector similarity measure are additionally explored. These similarity measures (SM) are applied to the environment of pattern recognition and medical diagnosis to assess the capability and feasibility of the interpreted measures. We additionally solved some numerical examples utilizing the established measures. We examine the dependability and validity of the proposed measures by comparing them with some existing measures. The advantages, comparative analysis, and graphical portrayal of the investigated interpreted measures and existing measures are additionally described in detail.
\end{abstract}

\section{Introduction}

Since fuzzy set [1] was presented, various extensions of this concept have been proposed, for example, interval-valued fuzzy set [2-4], intuitionistic fuzzy set [5], interval-valued intuitionistic fuzzy set [6], linguistic fuzzy set [7], type-2 fuzzy set [8], type- $n$ fuzzy set [8], and fuzzy multiset [9]. Baležentis and Zeng [10] interpreted group multicriteria decision making based upon an interval-valued fuzzy number. Su et al. [11] explored the intuitionistic fuzzy decision making with SMs and OWA operator. Peng et al. [12] described some intuitionistic fuzzy weighted geometric distance measures and their application to group decision making. An intuitionistic fuzzy MULTIMOORA approach for multicriteria assessment of the energy storage technologies was described by Zhang et al. [13]. Zhou et al. [14] interpreted the normalized weighted Bonferroni harmonic mean-based intuitionistic fuzzy operators and their application to the sustainable selection of search and rescue robots. An interval-valued intuitionistic fuzzy multiple attribute decision making based on nonlinear programming methodology and TOPSIS method was described by Zeng et al. [15]. The theory of linear Diophantine fuzzy set (LDFS) was explored by Riaz and Hashmi [16]. The proposed model has a resemblance with the well-known linear Diophantine equation $a x+b y=c$ in the number theory. Since IFSs, PFSs, and q-ROFSs have some limitations on membership/nonmembership grades. In order to get rid of such limitations, the theory of LDFS was explored with the addition of reference parameters. This idea removes the restrictions of membership/nonmembership grades and the decision maker can freely choose the grades without any limitation. 
This structure also categorizes the problem by choosing different types of reference parameters. The structure of LDFS and its graphical representation explaining these concepts with the help of illustrations are given in reference [16]. In the real world, we often encounter fuzzy situations in which it is hard to determine the membership of an element to a set because of the doubts between a few different values. To resolve this sort of issue, Torra [17] presented the idea of the hesitant fuzzy set (HFS), which allows the membership to have a set of possible finite values. Since its appearance, more and more multiple decision-making theories and methods have been proposed under a hesitant fuzzy environment [18-21]. Xu and Xia [22] described the distance measures for HFS and compared similarity measures. Distance and SMs among HFSs and their application in pattern recognition were expressed by Zeng et al. [23]. Mu et al. [24] described a novel aggregation principle for hesitant fuzzy elements. Decomposition theorems and extension principles for HFSs are explored by Alcantud and Torra [25]. Liao and $\mathrm{Xu}$ [26] described subtraction and division operation over HFSs. Bisht and Kumar [27] characterized the fuzzy time series forecasting method based on HFSs. Alcantud and Giarlotta [28] proposed the expansion of Torra's idea of HFSs. Novel distance and SMs on HFSs with application to clustering analysis were introduced by Zhang and $\mathrm{Xu}$ [29]. Farhadinia and Herrera-Viedma [30] described multiple criteria group decision-making methods based on extended HFSs with unknown weight information. Chen et al. [31] interpreted the idea of interval-valued hesitant fuzzy sets (IvHFSs) which is the generalization of HFS.

Dual hesitant fuzzy set (DHFS), as another augmentation of HFS, was proposed by Zhu et al. [32], in which the participation degree and nonenrollment level of a component to a given set are meant by two sets of a few fresh qualities. It includes a fuzzy set, fuzzy multiset, intuitionistic fuzzy set, and hesitant fuzzy set as uncommon cases and has gotten increasingly more consideration from scientists as of late. Wang et al. [33] characterized distance and comparability proportions of DHFSs with their applications to different quality dynamics. Zhu and Xu [34] characterized a few outcomes for DHFSs. Tyagi [35] introduced the relationship coefficient of DHFS and its application. Considering such capacities gives us more praiseworthy and adaptable access to allocate esteems for every component in the space. Obviously, DHFSs can mirror the human's reluctance more dispassionately than the other existing augmentations of the fuzzy set (AIFSs, IVAIFSs, HFSs, and so on.). In any case, in the soul of what has been accomplished for IVFSs, Farhadinia [36] presented a dual interval-valued hesitant fuzzy set (DIVHFS) where its principal trademark is that the value of the membership and nonmembership function are set of intervals as opposed to a set of definite numbers. Many scholars raised the question, what happened when a decision-maker changes the range of the fuzzy set into a unit disc. Ramot et al. [37] presented the possibility of complex FS (CFS), which contains membership grade as a complex number of a unit disc in the complex plane. CFS manages two dimensions in a solitary set. CFS is an amazing strategy to outline the conviction of a person in the development of evaluations. Many scholars have work on multiattribute decision making [38-44].

In real-life issues, we run over various conditions where we need to measure the weakness existing in the data to choose perfect decisions. Vector similarity measures (VSMs) and hybrid vector similarity measure are significant apparatuses for dealing with dubious data present in our day-today life problems. Different measures, for instance, similarity, exponential, separation, entropy, and incorporation process the flawed information and engage us to show up at some goal. Starting late, these measures have expanded a great deal of thought from various makers due to their wide applications in various fields, for instance, pattern recognition, medical diagnosis, and decision making. All the current techniques of chiefs, considering VSM and hybrid vector similarity measures, in FS, CFS, and HFS speculations, manage participation capacities having a place with a unit span as a subset in the idea of HFS. In the CDHFS speculation, membership and nonmembership degrees are astoundingly regarded and are addressed in polar ways. These all thoughts worked feasibly, in any case, when a pioneer stood up to such kinds of information which contains two-dimensional information in a singular set. For instance, $\left\{\left\{0.8 e^{i 2 \pi(0.4)}, 0.5 e^{i 2 \pi(0.7)}\right\},\left\{0.2 e^{i 2 \pi(0.2)}, 0.1 e^{i 2 \pi(0.5)}\right\}\right.$, $\left.\left\{0.15 e^{i 2 \pi(0.35)}, 0.7 e^{i 2 \pi(0.56)}\right\}\right\}$ at that point all the current ideas are fizzled. For adapting to such sorts of issues, the CDHFS is a capable method to resolve realistic decision problems in the environment of the FS theory. CDHFS is more impressive and broader than existing ideas such as HFS, CFS, and FS to adapt to awkward and confusing data, in realworld decisions. Since these all thoughts are the particular cases of the investigated CDHFS, the upsides of the introduced CDHFS are talked about underneath:

(1) At the point when we pick the imaginary parts of the CDHFS as zero, the CDHFS is diminished into DHFS which is as $\{\{0.8,0.5\},\{0.2,0.1\},\{0.15,0.7\}\}$

(2) At the point when we pick the CDHFS as a singleton set, the CDHFS is diminished into CIFS which is as $\left\{0.8 e^{i 2 \pi(0.4)}, 0.5 e^{i 2 \pi(0.7)}\right\}$

(3) At the point when we pick the CDHFS as a singleton set and the imaginary parts as zero, the CDHFS is decreased into IFS which is as $\{0.8,0.5\}$

The theory of CDHFS is a proficient technique to cope with uncertain and awkward information in the real-life problems. CDHFS contains the grade of membership and the grade of nonmembership in the form of complex number belonging to the complex plane in a unit disc, whose real part and imaginary part are the subset of unit interval. CDHFS is more generalized than many existing notions such as fuzzy sets, intuitionistic fuzzy sets, hesitant fuzzy sets, dual hesitant fuzzy sets, complex fuzzy sets, complex intuitionistic fuzzy sets, and complex hesitant fuzzy sets . For example, if we choose the value of the imaginary part to be zero, then the CDHFS is converted for dual hesitant fuzzy sets, and if we choose the value of nonmembership to be zero, then the CDHFS is converted for HFS. Similarly, if we choose the value of nonmembership to be zero, then the 
explored approach is converted for complex hesitant fuzzy sets. Inspired by the above difficulties and maintaining the benefits of the CDHFS, in this paper, some key contributions are made as follows:

(1) Complex dual hesitant fuzzy set (CDHFS) is a mix of two alterations, called complex fuzzy set (CFS) and dual hesitant fuzzy set (DHFS). CDHFS makes two degrees, called membership valued and nonmembership valued in the form of subset of a unit disc in a complex plane, and is a capable method to adapt to questionable and capricious real-world issues. The aim of the paper is to investigate the idea of a CDHFS and its operational laws.

(2) The novel approach of CIvDHFS and its essential laws are investigated and advocated with the assistance of an example.

(3) Further, the VSMs, WVSMs, hybrid VSM, and weighted hybrid VSM and their significant attributes are likewise investigated.

(4) These SMs are applied to the environment of pattern recognition and medical diagnosis to assess the capability and plausibility of the interpreted measures. We likewise illuminated some numerical examples utilizing the interpreted measures.

(5) We examine the dependability and legitimacy of the proposed measures by contrasting them with existing measures.

(6) The advantages, relative investigation, and graphical portrayal of the investigated measures and existing measures are additionally talked about in detail.

The rest of the paper is organized as follows: in Section 2, we review some basic definitions such as FS, CFS, HFS, DHFS, and IvDHFS. In Section 3, we investigate the thought of a CDHFS and its operational laws. The epic methodology of CIvDHFS and its basic laws are additionally investigated and defended with the assistance of models. In Section 4, the vector similarity measures (VSMs), weighted vector similarity measures (WVSMs), hybrid vector similarity measure, and weighted hybrid vector similarity measure are investigated. In Section 5, these measures are applied to the environment of pattern recognition and medical diagnosis to assess the capability and practicality of the explored measures. We likewise tackled some numerical models utilizing the explored measures. We examine the dependability and legitimacy of the proposed measures by contrasting them with existing measures given in Section 6. The points of interest, relative investigation, and graphical portrayal of the investigated gauges and existing measures are likewise talked about in detail. Section 7 concludes the paper.

\section{Preliminaries}

In this section, we survey fundamental definitions such as FS, CFS, HFS, DHFS, and IvDHFS. Through this article, $X$ speaks to a fix set.
Definition 1 (see [1])A FS $\mathcal{S}$ is of the structure:

$$
\mathcal{S}=\left\{\left(x, \mu_{\mathcal{S}}(x)\right) \mid x \in X\right\},
$$

with a condition $0 \leq \mu_{\delta}(x) \leq 1$, where $\mu_{\delta}(x)$ represents the grade of truth. Through this article, the collection of all FSs on $X$ is represented by $F \mathcal{S}(X)$. The pair $\mathcal{S}=\left(x, \mu_{\mathcal{S}}(x)\right)$ is known as fuzzy number (FN).

Definition 2 (see [37])A CFS $\mathcal{S}$ is of the structure:

$$
\mathcal{S}=\left\{\left(x, \mu_{\mathcal{S}}(x)\right) \mid x \in X\right\},
$$

where $\mu_{\mathcal{S}}(x)=\gamma_{\mathcal{S}}(x) \cdot e^{i 2 \pi\left(\omega_{\gamma_{S}}(x)\right)}$ represents the complexvalued truth grade in the form of polar coordinate, where $\gamma_{\delta}(x), \omega_{\gamma_{\delta}}(x) \in[0,1]$. Additionally, the pair $\left(x, \gamma_{\delta}(x)\right.$. $\left.e^{i 2 \pi\left(\omega_{\gamma_{\delta}}(x)\right)}\right)$ is known as complex fuzzy number (CFN).

Definition 3 (see [17])An HFS $\mathcal{S}$ is of the structure:

$$
\mathcal{S}=\left\{\left(x, \mu_{\mathcal{S}}(x)\right) \mid x \in X\right\},
$$

where $\mu_{\delta}(x)$ is the set of different finite values in $[0,1]$ representing the grade of truth for each element $x \in X$. Further, the pair $\mathcal{S}=\left(x, \mu_{\mathcal{S}}(x)\right)$ is known as hesitant fuzzy number (HFN).

Definition 4 (see [32])A DHFS $\mathcal{S}$ is of the structure:

$$
\mathcal{S}=\left\{\left(x,\left(\mu_{\mathcal{S}}(x), v_{\mathcal{\delta}}(x)\right)\right) \mid x \in X\right\},
$$

where $\mu_{\delta}(x)$ and $v_{\delta}(x)$ are two finite subsets in $[0,1]$, representing the membership grade and nonmembership grade of the component $x \in X$, respectively, with the conditions $0 \leq \gamma_{\mathcal{S}}(x), \delta_{\mathcal{S}}(x) \leq 1,0 \leq \gamma_{\mathcal{S}}^{+}(x)+\delta_{\delta}^{+}(x) \leq 1$, where $\gamma_{\delta}(x) \in \mu_{\delta}(x), \delta_{\delta}(x) \in v_{\mathcal{S}}(x), \gamma_{\mathcal{S}}^{+}(x) \in \mu_{\delta}^{+}(x)=\cup_{\gamma_{\delta}(x) \epsilon}$ $\mu_{\delta}(x) \max \left\{\gamma_{\delta}(x)\right\}$, and $\delta_{\delta}^{+}(x) \in v_{\delta}^{+}(x)=\cup_{\delta_{\delta}(x) \in v_{\delta}(x)}$ $\max \left\{\delta_{\delta}(x)\right\}$.

Definition 5 (see [33])For any two DHFSs $\mathcal{S}$ and $\mathscr{T}$, the similarity measure $\mathbb{S}(\mathcal{S}, \mathscr{T})$ satisfies the following axioms:
(1) $0 \leq \mathbb{S}(\mathcal{S}, \mathscr{T}) \leq 1$
(2) $\mathbb{S}(\mathcal{S}, \mathscr{T})=1 \Longleftrightarrow \mathcal{S}=\mathscr{T}$
(3) $\mathbb{S}(\mathcal{S}, \mathscr{T})=\mathbb{S}(\mathscr{T}, \mathcal{S})$

Definition 6 (see [33])For any two DHFSs $\mathcal{S}$ and $\mathscr{T}$, the distance measure $\mathbb{D}(\mathcal{S}, \mathscr{T})$ satisfies the following axioms:

(1) $0 \leq \mathbb{D}(\mathcal{S}, \mathscr{T}) \leq 1$

(2) $\mathbb{D}(\mathcal{S}, \mathscr{T})=1 \Longleftrightarrow \mathcal{S}=\mathscr{T}$

(3) $\mathbb{D}(\mathcal{S}, \mathscr{T})=\mathbb{D}(\mathscr{T}, \mathcal{S})$

From the above discussion, we obtain that $\mathbb{S}(\mathcal{S}, \mathscr{T})=1-\mathbb{D}(\mathcal{S}, \mathscr{T})$.

Definition 7 (see [36])An IvDHFS $\delta$ is of the structure:

$$
\mathcal{\delta}=\left\{\left(x,\left(\mu_{\mathcal{\delta}}(x), v_{\mathcal{\delta}}(x)\right)\right) \mid x \in X\right\},
$$


where $\mu_{\mathcal{\delta}}(x)=U_{\left[\gamma_{\delta}^{L}(x) \gamma_{\delta}^{U}\right] \in \mu_{\mathcal{S}}(x)} \max \left\{\left[\gamma_{\mathcal{S}}^{L}(x), \gamma_{\mathcal{S}}^{U}(x)\right]\right\}$ and $v_{\mathcal{S}}(x)=\cup_{\left[\delta_{\delta}^{L}(x) \delta_{\delta}^{U}\right] \in v_{\mathcal{S}}(x)} \max \left\{\left[\delta_{\delta}^{L}(x), \delta_{\mathcal{S}}^{U}(x)\right]\right\}$ are two finite subsets of some interval values in $[0,1]$, representing the membership grade and nonmembership grade of the component $x \in X$, respectively, with the conditions $\left[\gamma_{\delta}^{L}(x) \gamma_{\delta}^{U}(x)\right],\left[\delta_{\delta}^{L}(x), \delta_{\delta}^{U}(x)\right] \subseteq[0,1]$, and $0 \leq\left(\gamma_{\delta}^{U}(x)\right)^{+}+$ $\left(\delta_{\delta}^{U}(x)\right)^{+} \leq 1$, where $\left[\gamma_{\delta}^{L}(x) \gamma_{\delta}^{U}(x)\right] \in \mu_{\delta}(x), \quad\left[\delta_{\delta}^{L}(x)\right.$, $\left.\delta_{\delta}^{U}(x)\right] \epsilon \quad v_{\mathcal{S}}(x), \quad\left(\gamma_{\mathcal{S}}^{U}(x)\right)^{+} \in \mu_{\mathcal{S}}^{+}(x)=\cup_{\left[\gamma_{\delta}^{L}(x) \gamma_{\delta}^{U}\right] \in \mu_{\mathcal{S}}(x)}$ $\max \left\{\gamma_{\delta}^{L}(x)\right\}$, and $\quad\left(\delta_{\delta}^{U}(x)\right)^{+} \in v_{\mathcal{\delta}}^{+}(x)=U_{\left[\delta_{\delta}^{L}(x), \delta_{\delta}^{U}\right] \in v_{\delta}(x)}$ $\max \left\{\delta_{\mathcal{S}}(x)\right\}$ for all $x \in X$.

\section{Complex Dual Hesitant Fuzzy Sets and Complex Interval-Valued Dual Hesitant Fuzzy Sets}

In this section, we have two subsections in which we defined the idea of CDHFS, CIvDHFS, and their operational laws.

3.1. Complex Dual Hesitant Fuzzy Sets. In this section, we investigated the notion of CDHFS which is the alteration of CFS and DHFS. We additionally investigated its operational laws.

Definition 8. A CDHFS $\delta$ is of the structure:

$$
\mathcal{S}=\left\{\left(x,\left(\mu_{\mathcal{\delta}}(x), v_{\mathcal{S}}(x)\right)\right) \mid x \in X\right\},
$$

where $\mu_{\delta}(x)=\left\{\left(x, \gamma_{\mathcal{S}_{p}}(x) \cdot e^{i 2 \pi\left(\omega_{\gamma_{\delta_{p}}}\right)}\right), p=1,2,3, \ldots, g\right\}$ and $v_{\mathcal{\delta}}(x)=\left\{\left(x, \delta_{\delta_{q}}(x) \cdot e^{i 2 \pi\left(\omega_{\gamma_{\delta_{q}}}\right)}, q=1,2,3, \ldots, h\right\}\right.$ represented the complex-valued membership grade and nonmembership grade, which are subsets of a unit disc in the complex plane with conditions $\gamma_{\delta_{p}}(x), \omega_{\gamma_{\delta_{p}}}(x), \delta_{\delta_{q}}(x)$, $\bar{\omega}_{\delta_{\delta_{q}}}(x) \in[0,1], \quad 0 \leq \gamma_{\mathcal{S}}^{+}(x)+\delta_{\delta}^{+}(x) \leq 1$, and $0 \leq \omega_{\gamma_{\delta}}^{+}(x)+$ $\bar{\omega}_{\delta_{\delta}}^{+}(x) \leq 1$, where $\quad \gamma_{\delta}^{+}(x)=\cup_{\gamma_{\delta_{p}}(x) \in \mu_{\delta}(x)} \max \left\{\gamma_{\delta_{p}}(x)\right\}$, $\omega_{\gamma_{\delta}}^{+}(x)=\cup_{\omega_{\delta_{p}}(x) \in \mu_{\delta}(x)} \max \left\{\omega_{\gamma_{\delta_{p}}}(x)\right\}, \delta_{\delta}^{+}(x)=\cup_{\delta_{\delta_{q}}(x) \in v_{\delta}(x)}$ $\max \left\{\delta_{\delta_{q}}(x)\right\}$, and $\bar{\omega}_{\delta_{\delta}}^{+}(x)=\cup_{\bar{\omega}_{\delta_{\delta_{q}}}(x) \in v_{\delta}(x)} \max \left\{\bar{\omega}_{\delta_{\delta_{q}}}(x)\right\}$ for $p=1,2, \ldots, g$ and $q=1,2, \ldots, h$. Further, $\mathcal{S}=\left(x,\left(\gamma_{\delta_{p}}(x) \cdot e^{i 2 \pi\left(\omega_{\gamma_{p}}(x)\right)}, \delta_{\delta_{q}}(x) \cdot e^{i 2 \pi\left(\bar{\omega}_{\delta_{\delta_{q}}}(x)\right)}\right)\right)$ is called complex dual hesitant fuzzy number (CDHFN).

Definition 9. Let $\delta=\left(x, \gamma_{\mathcal{S}_{p}}(x) \cdot e^{i 2 \pi\left(\omega_{\gamma_{\delta_{p}}}(x)\right)}, \delta_{\mathcal{S}_{q}}(x)\right.$. $\left.e^{i 2 \pi\left(\bar{\omega}_{\delta_{\delta_{q}}}(x)\right)}\right) \quad$ and $\quad \mathscr{T}=\left(x, \gamma_{\mathscr{T}_{p}}(x) \cdot e^{i 2 \pi\left(\omega_{\gamma \mathscr{T}_{p}}(x)\right)}, \delta_{\mathscr{T}_{q}}(x)\right.$. $\left.e^{i 2 \pi\left(\bar{\omega}_{\mathscr{S}_{q}}(x)\right)}\right)$ be two CDHFNs. Then their complement, union, and intersection are defined as follows:

(1) $\mathcal{S}^{c}=\left\{\left(x,\left(v_{\mathcal{\delta}}(x), \mu_{\mathcal{\delta}}(x)\right)\right)\right\}$

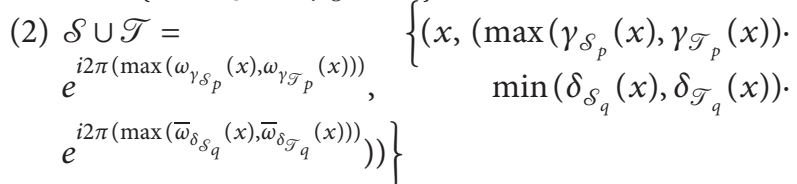

$$
\text { (3) } \begin{aligned}
& \mathcal{S} \cap \mathscr{T}=\left\{\left(x,\left(\min \left(\gamma_{\mathcal{S}_{p}}(x), \gamma_{\mathscr{F}_{p}}(x)\right) .\right.\right.\right. \\
& e^{i 2 \pi\left(\max \left(\omega_{\gamma_{\delta_{p}}}(x), \omega_{\gamma_{\mathcal{F}_{p}}}(x)\right)\right)}, \max \left(\delta_{\mathcal{S}_{q}}(x), \delta_{\mathscr{T}_{q}}(x)\right) . \\
& \left.\left.\left.e^{i 2 \pi\left(\max \left(\bar{\omega}_{\delta_{\delta_{q}}}(x), \bar{\omega}_{\delta_{\mathscr{J}_{q}}}(x)\right)\right)}\right)\right)\right\}
\end{aligned}
$$

Example 1. Let

$$
\mathcal{S}=\left\{\begin{array}{c}
\left(x_{1},\left\{\left\{\left\{0.7 e^{i 2 \pi(0.4)}, 0.3 e^{i 2 \pi(0.6)}\right\},\right.\right.\right. \\
\left.\left.\left.\left\{0.2 e^{i 2 \pi(0.2)}, 0.1 e^{i 2 \pi(0.3)}\right\}\right\}\right\}\right), \\
\left(x_{2},\left\{\left\{0.7 e^{i 2 \pi(0.6)}\right\},\left\{0.2 e^{i 2 \pi(0.3)}\right\}\right\}\right), \\
,\left\{\left\{0.6 e^{i 2 \pi(0.5)}, 0.5 e^{i 2 \pi(0.4)}, 0.4 e^{i 2 \pi(0.7)}\right\},\right. \\
\left.\left.\left\{0.3 e^{i 2 \pi(0.1)} 0.1 e^{i 2 \pi(0.2)}\right\}\right\}\right), \\
\left(x_{4},\left\{\left\{0.4 e^{i 2 \pi(0.5)}, 0.6 e^{i 2 \pi(0.8)}\right\},\left\{0.515 e^{i 2 \pi(0.1)}\right\}\right\}\right), \\
\left(x_{5},\left\{\left\{0.5 e^{i 2 \pi(0.6)}, 0.4 e^{i 2 \pi(0.5)}, 0.2 e^{i 2 \pi(0.4)}\right\},\right.\right. \\
\\
\left.\left.\left\{0.3 e^{i 2 \pi(0.2)}, 0.1 e^{i 2 \pi(0.2)}, 0.2 e^{i 2 \pi(0.3)}\right\}\right\}\right)
\end{array}\right\}
$$

and

$$
\mathscr{T}=\left\{\begin{array}{c}
\left(x_{1},\left\{\left\{0.3 e^{i 2 \pi(0.2)}\right\}\left\{0.65 e^{i 2 \pi(0.7)}, 0.5 e^{i 2 \pi(0.6)}\right\}\right\}\right), \\
\left(x_{2},\left\{\left\{0.8 e^{i 2 \pi(0.6)}, 0.55 e^{i 2 \pi(0.55)}, 0.45 e^{i 2 \pi(0.8)}\right\},\right.\right. \\
\left.\left.\left\{0.1 e^{i 2 \pi(0.15)}, 0.15 e^{i 2 \pi(0.1)}, 0.1 e^{i 2 \pi(0.5)}\right\}\right\}\right), \\
\left(x_{3},\left\{\left\{0.3 e^{i 2 \pi(0.55)}\right\},\left\{0.25 e^{i 2 \pi(0.35)}\right\}\right\}\right), \\
\left(x _ { 4 } \left\{\left\{0.35 e^{i 2 \pi(0.65)}, 0.65 e^{i 2 \pi(0.5)}\right\},\right.\right. \\
\left.\left.\left\{0.15 e^{i 2 \pi(0.25)}, 0.3 e^{i 2 \pi(0.3)}\right\}\right\}\right), \\
\left(x_{5},\left\{\left\{0.5 e^{i 2 \pi(0.3)}, 0.3 e^{i 2 \pi(0.6)}\right\},\left\{0.2 e^{i 2 \pi(0.25)}\right\}\right\}\right)
\end{array}\right\}
$$

be two CDHFSs. Then their operational laws are given as follows:

(1)

$$
\mathcal{S}^{c}=\left\{\begin{array}{c}
\left(x_{1},\left\{\left\{\left\{0.2 e^{i 2 \pi(0.2)}, 0.1 e^{i 2 \pi(0.3)}\right\},\right.\right.\right. \\
\left.\left.\left\{0.7 e^{i 2 \pi(0.4)}, 0.3 e^{i 2 \pi(0.6)}\right\}\right\}\right\}, \\
\left(x_{2},\left\{\left\{0.2 e^{i 2 \pi(0.3)}\right\},\left\{0.7 e^{i 2 \pi(0.6)}\right\}\right\}\right), \\
\left(x_{3},\left\{\left\{0.3 e^{i 2 \pi(0.1)}, 0.1 e^{i 2 \pi(0.2)}\right\},\right.\right. \\
\left.\left.\left\{0.6 e^{i 2 \pi(0.5)}, 0.5 e^{i 2 \pi(0.4)}, 0.4 e^{i 2 \pi(0.7)}\right\}\right\}\right), \\
\left(x,\left\{\left\{0.15 e^{i 2 \pi(0.1)}\right\},\left\{0.4 e^{i 2 \pi(0.5)}, 0.6 e^{i 2 \pi(0.8)}\right\}\right\}\right) \\
\left(x_{5},\left\{\left\{0.3 e^{i 2 \pi(0.2)}, 0.2 e^{i 2 \pi(0.3)}\right\},\right.\right. \\
\\
\left.\left.\left\{0.5 e^{i 2 \pi(0.6)}, 0.4 e^{i 2 \pi(0.5)}, 0.2 e^{i 2 \pi(0.4)}\right\}\right\}\right)
\end{array}\right\}
$$


(2) $\mathcal{S} \cup \mathscr{T}=$

$\left\{\begin{array}{l}\left(x_{1},\left\{\left\{\left\{0.7 e^{i 2 \pi(0.4)}, 0.3 e^{i 2 \pi(0.1)}\right\},\left\{0.2 e^{i 2 \pi(0.2)}, 0.1 e^{i 2 \pi(0.3)}\right\}\right\}\right\}\right) \\ \left(x_{2},\left\{\left\{0.8 e^{i 2 \pi(0.6)}, 0.55 e^{i 2 \pi(0.55)}, 0.45 e^{i 2 \pi(0.8)}\right\},\left\{0.1 e^{i 2 \pi(0.15)}\right\}\right\}\right), \\ \left(x_{3},\left\{\left\{0.6 e^{i 2 \pi(0.55)}, 0.5 e^{i 2 \pi(0.4)}, 0.4 e^{i 2 \pi(0.7)}\right\},\left\{0.25 e^{i 2 \pi(0.1)}\right\}\right\}\right), \\ \left(x_{4}\left\{\left\{0.4 e^{i 2 \pi(0.65)}, 0.65 e^{i 2 \pi(0.8)}\right\},\left\{0.15 e^{i 2 \pi(0.1)}\right\}\right\}\right) \\ \left(x_{5},\left\{\left\{0.5 e^{i 2 \pi(0.6)}, 0.4 e^{i 2 \pi(0.6)} 0.2 e^{i 2 \pi(0.4)}\right\},\left\{0.2 e^{i 2 \pi(0.2)}\right\}\right\}\right)\end{array}\right\}$

(3) $\mathcal{S} \cap \mathscr{T}=$

$\left\{\begin{array}{l}\left(x_{1},\left\{\left\{0.3 e^{i 2 \pi(0.2)}\right\}\left\{0.65 e^{i 2 \pi(0.7)}, 0.5 e^{i 2 \pi(0.6)}\right\}\right\}\right), \\ \left(x_{2},\left\{\left\{0.7 e^{i 2 \pi(0.6)}\right\},\left\{0.2 e^{i 2 \pi(0.3)}, 0.15 e^{i 2 \pi(0.1)}, 0.1 e^{i 2 \pi(0.5)}\right\}\right\}\right), \\ \left(x_{3},\left\{\left\{0.45 e^{i 2 \pi(0.5)}\right\},\left\{0.3 e^{i 2 \pi(0.35)}, 0.1 e^{i 2 \pi(0.2)}\right\}\right\}\right), \\ \left(x_{4},\left\{\left\{0.35 e^{i 2 \pi(0.5)}, 0.6 e^{i 2 \pi(0.5)}\right\},\left\{0.15 e^{i 2 \pi(0.25)}, 0.3 e^{i 2 \pi(0.3)}\right\}\right\}\right) \\ \left(x_{5},\left\{\left\{0.5 e^{i 2 \pi(0.3)}, 0.3 e^{i 2 \pi(0.5)}\right\},\right.\right. \\ \left.\left.\left\{0.3 e^{i 2 \pi(0.25)}, 0.1 e^{i 2 \pi(0.2)}, 0.2 e^{i 2 \pi(0.3)}\right\}\right\}\right)\end{array}\right\}$

3.2. Complex Interval-Valued Dual Hesitant Fuzzy Sets. In this section, we investigated the notion of CIvDHFS and additionally investigated its operational laws.

Definition 10. A CIvDHFS $\mathcal{S}$ is of the structure:

$$
\mathcal{S}=\left\{\left(x,\left(\mu_{\mathcal{S}}(x), v_{\mathcal{S}}(x)\right)\right) \mid x \in X\right\},
$$

where

$$
\begin{aligned}
\mu_{\mathcal{S}}(x)= & {\left[\mu_{\mathcal{S}}^{L}(x), \mu_{\mathcal{S}}^{U}(x)\right] } \\
= & \left\{\left(x,\left[\gamma_{\mathcal{S}_{p}}^{L}(x), \gamma_{\mathcal{S}_{p}}^{U}(x)\right] \cdot e^{i e \pi\left(\left[\omega_{\gamma_{\mathrm{p}}}^{L}(x), \omega_{\gamma_{\delta_{p}}}^{U}(x)\right]\right)}\right),\right. \\
& p=1,2,3, \ldots, g\}, \\
v_{\mathcal{S}}(x)= & {\left.\left.\left[v_{\mathcal{S}}^{L}(x), v_{\mathcal{S}}^{U}(x)\right]\right\}\right) } \\
= & \left\{\left(x,\left[\delta_{\mathcal{S}_{q}}^{L}(x), \delta_{\delta_{q}}^{U}(x)\right] \cdot e^{i e \pi\left(\left[\bar{\omega}_{\delta_{\delta_{q}}}^{L}(x), \bar{\omega}_{\delta_{\delta_{q}}}^{U}(x)\right]\right)},\right.\right. \\
& p=1,2,3, \ldots, h\},
\end{aligned}
$$

represented the complex-valued membership grade and nonmembership grade, which are finite subsets of different interval values of a unit disc in complex plane with conditions $\gamma_{\delta_{p}}^{L}(x), \gamma_{\delta_{p}}^{U}(x), \omega_{\gamma_{\delta_{p}}}^{L}(x), \omega_{\gamma_{\delta_{p}}}^{U}(x), \delta_{\delta_{q}}^{L}(x), \delta_{\delta_{q}}^{U}(x)$, $\omega_{\gamma_{\delta_{p}}}^{L}(x), \omega_{\gamma_{\delta_{p}}}^{U}(x) \in[0,1], 0 \leq\left(\gamma_{\mathcal{S}}^{U}(x)\right)^{+}+\left(\delta_{\delta}^{U}(x)\right)^{+} \leq 1$, and $0 \leq\left(\omega_{\gamma_{\mathcal{S}}}^{U}(x)\right)^{+}+\left(\Phi_{\delta_{\mathcal{S}}}^{U}(x)\right)^{+} \leq 1, \quad$ where $\quad\left(\gamma_{\mathcal{S}}^{U}(x)\right)^{+}=$ $\cup_{\left[\gamma_{\delta_{p}}^{L}(x), \gamma_{\delta_{p}}^{U}(x)\right] \in \mu_{\mathcal{S}}(x)} \max \left\{\gamma_{\mathcal{S}_{p}}^{L}(x)\right\}, \quad\left(\omega_{\gamma_{\delta}}^{U}(x)\right)^{+}=$ $\cup_{\left[\omega_{\gamma_{\delta_{p}}}^{L}(x), \omega_{\gamma_{\delta_{p}}}^{U}(x)\right] \in \mu_{\mathcal{\delta}}(x)} \max \left\{\omega_{\gamma_{\delta_{p}}^{U}}^{U}(x)\right\},\left(\delta_{\mathcal{\delta}}^{U}(x)\right)^{U}=\mathrm{U}_{\left[\delta_{\delta_{q}}^{L}(x), \gamma_{\delta_{q}}^{U}\right.}$ $(x)] \in v_{\mathcal{S}}(x) \max \left\{\delta_{\delta_{p}}^{L}(x)\right\}, \quad$ and $\quad\left(\varpi_{\delta_{\delta}}^{U}(x)\right)^{+}=$

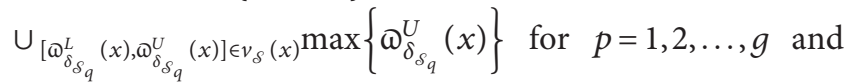
$q=1,2, \quad \ldots, h . \quad$ Further, $\quad\left(\left[\gamma_{\delta_{p}}^{L}, \gamma_{\delta_{p}}^{U}\right] \cdot e^{i 2 \pi\left(\left[\omega_{\gamma_{\delta_{p}}}^{L}(x), \omega_{\gamma_{\delta_{p}}}^{U}\right]\right)}\right.$, $\left.\left[\delta_{\delta_{q}}^{L}(x), \delta_{\delta_{q}}^{U}\right] \cdot e^{i 2 \pi\left(\left[\omega_{\delta_{\delta_{q}}}^{L}(x), \omega_{\delta_{\delta_{q}}^{U}}\right]\right)}\right)$ is called complex intervalvalued dual hesitant fuzzy number (CIvDHFN).

Definition 11. Let $\mathcal{S}=\left(x,\left(\left[\gamma_{\mathcal{S}_{p}}^{L}, \gamma_{\mathcal{S}_{p}}^{U}\right] \cdot e^{i 2 \pi\left(\left[\omega_{\gamma_{\delta_{p}}}^{L}(x), \omega_{\gamma_{\delta_{p}}}^{U}\right]\right)}\right.\right.$, $\left.\left[\delta_{\delta_{q}}^{L}(x), \delta_{\delta_{q}}^{U}\right] \cdot e^{i 2 \pi\left(\left[\omega_{\delta_{\delta_{q}}}^{L}(x), \Theta_{\delta_{\delta_{q}}^{U}}^{U}\right)\right)}\right)$ and $\mathscr{T}=\left(x,\left(\left[\gamma_{\mathscr{T}_{p}}^{L}, \gamma_{\mathscr{T}_{p}}^{U}\right] \cdot\right.\right.$ $e^{i 2 \pi\left(\left[\omega_{\gamma_{T_{p}}}^{L}(x), \omega_{\gamma_{T_{p}}}^{U}\right]\right)},\left[\delta_{\mathscr{T}_{q}}^{L}(x), \delta_{\mathscr{T}_{q}}^{U}\right] \cdot e^{i 2 \pi\left(\left[\omega_{\delta_{\mathscr{T}_{q}}^{L}}^{L}(x), \omega_{\delta_{\mathscr{T}_{q}}^{U}}^{U}\right)\right)}$ be two CIvDHFNs. Then their complement, union, and intersection are defined as follows:

(1) $\mathcal{S}^{c}=\left\{\left(x,\left(\left(v_{\mathcal{S}}\right), \mu_{\mathcal{S}}(x)\right)\right)\right\}$

(2) $\mathcal{S} \cup \mathscr{T}=$

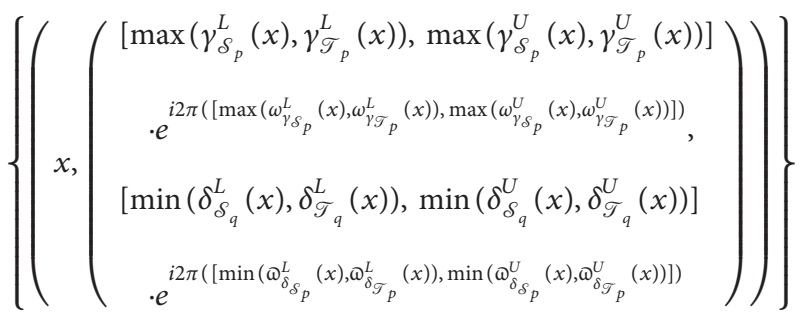

(3) $\mathcal{S} \cap \mathscr{T}=$

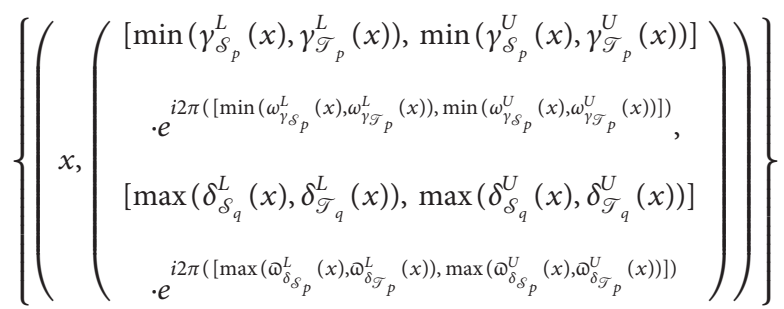


Example 2. Let

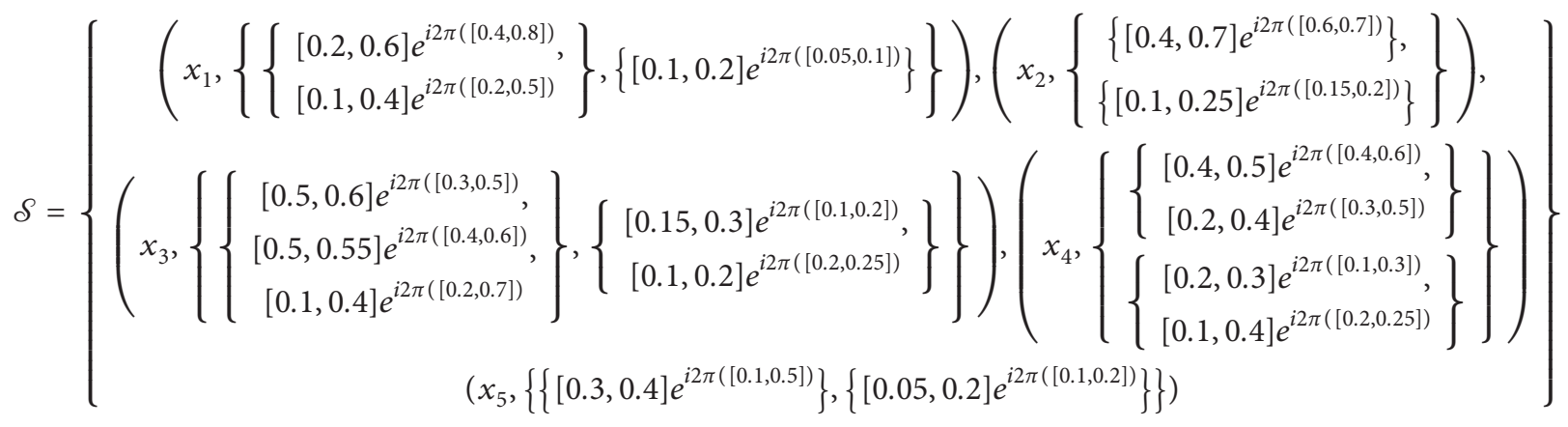

and

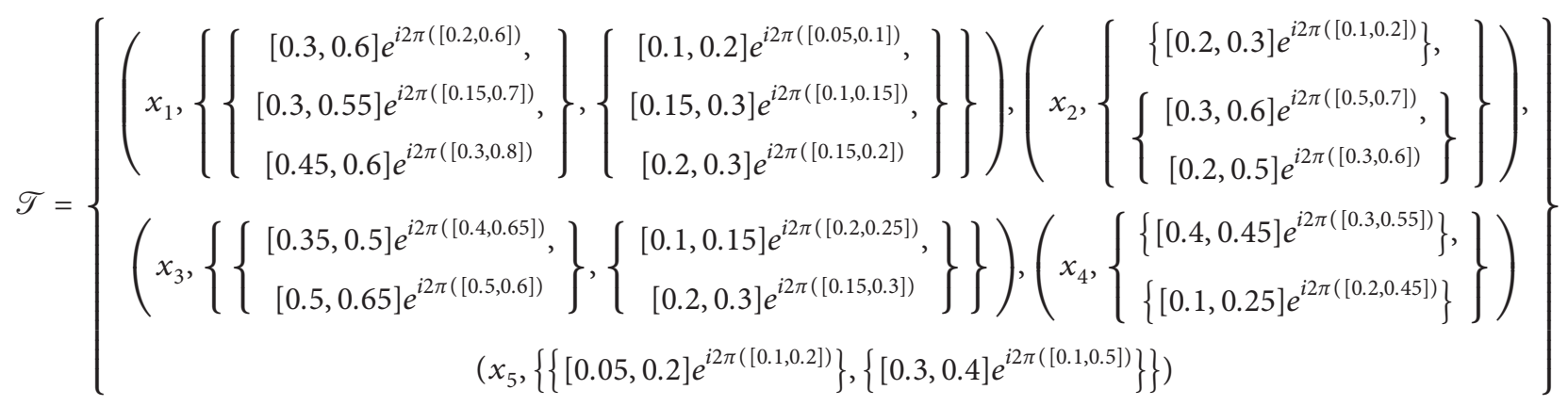

be two CIvDHFSs. Then its operational laws are given as follows:

(1)

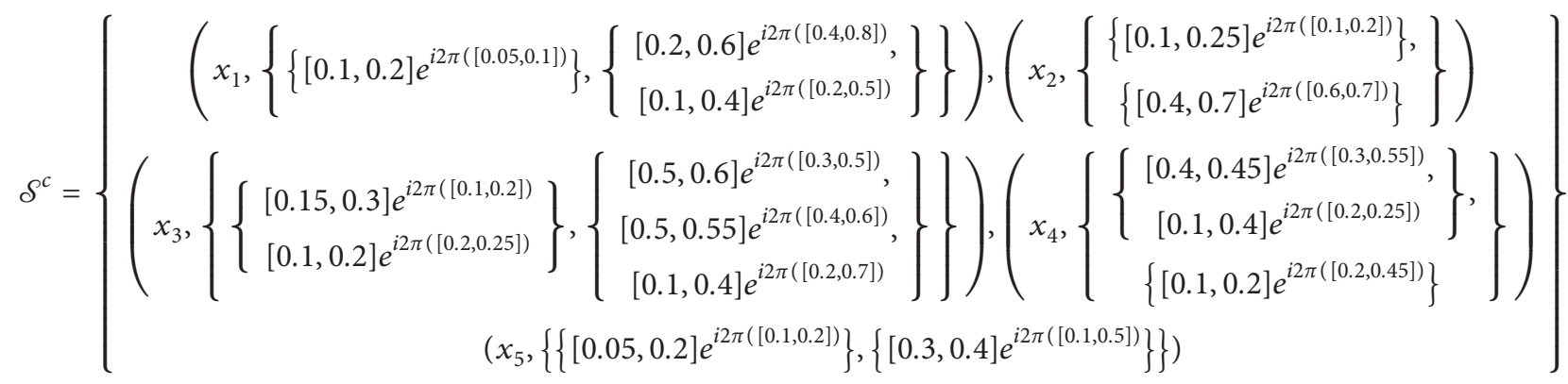


(2)

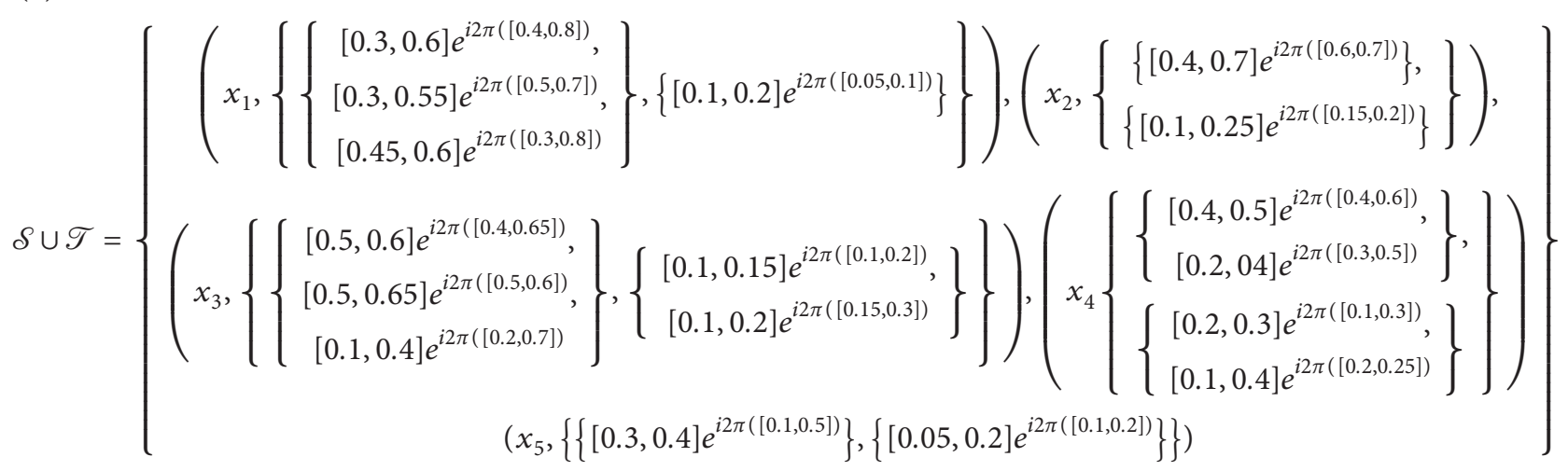

(3)

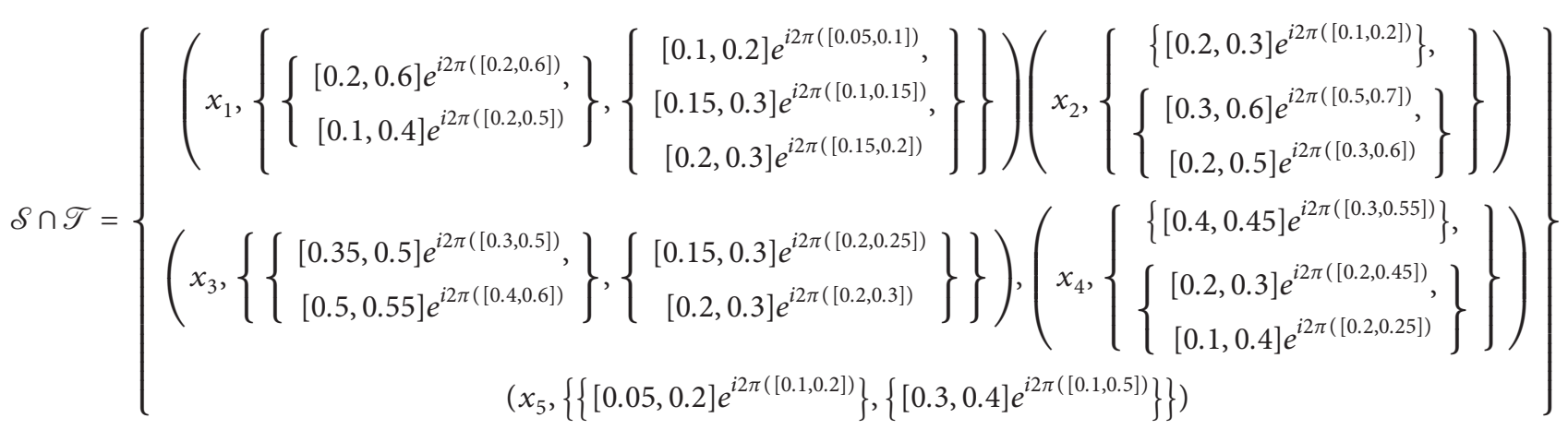

\section{The Similarity Measures For CDHFSs}

The theory of similarity measures (SM) is an essential idea of the human point of view. SM assumes a significant job in numerous fields, for example, machine learning and decision making. In this section, we have two subsections, firstly, vector similarity measures (VSMs) and secondly, hybrid vector similarity measure.
4.1. Vector Similarity Measures of CDHFSs. The VSM is one of the important tools for the similarity degree between objects. We straightforwardly utilized Jaccard, Dice, and Cosine SM. Presently, in this segment, we characterize VSMs and weighted VSMs (WVSMs) for CDHFSs.

Definition 12. Suppose that $\mathcal{S}$ and $\mathscr{T}$ are two CDHFSs on $X$, then the Jaccard similarity measure (JSM) between $\mathcal{S}$ and $\mathscr{T}$ is denoted and defined as follows:

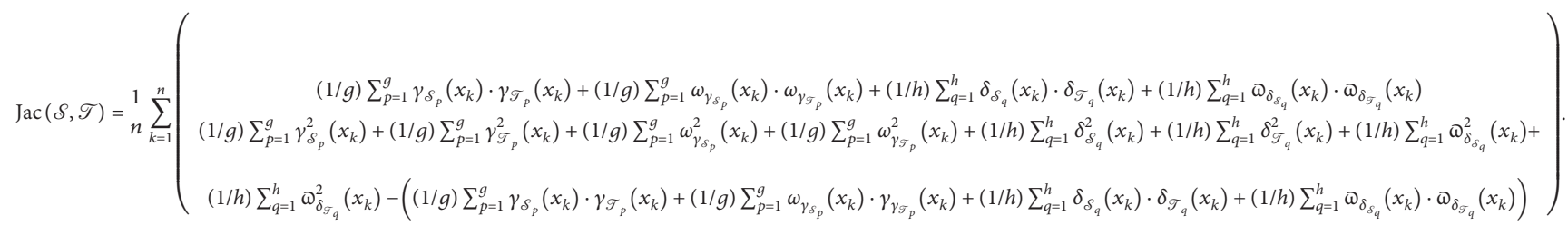

JSMs fulfill the following axioms:

(1) $0 \leq \operatorname{Jac}(\mathcal{S}, \mathscr{T}) \leq 1$
(2) $\operatorname{Jac}(\mathcal{S}, \mathscr{T})=\operatorname{Jac}(\mathscr{T}, \mathcal{S})$

(3) $\operatorname{Jac}(\mathcal{S}, \mathscr{T})=1$, if $\mathcal{S}=\mathscr{T}$ 
Theorem 1. Prove that equation (9) holds the above three conditions.

Proof

(1) Since $\quad(1 / g) \sum_{p=1}^{g} \gamma_{\mathcal{S}_{p}}\left(x_{k}\right) \cdot \gamma_{\mathcal{T}_{p}}\left(x_{k}\right) \in[0,1], \quad(1 / g)$ $\sum_{p=1}^{g} \omega_{\gamma_{\delta_{p}}}\left(x_{k}\right) \cdot \omega_{\gamma_{T_{p}}}\left(x_{k}\right) \in[0,1],(1 / h) \sum_{q=1}^{h} \delta_{\mathcal{S}_{q}}\left(x_{k}\right)$. $\delta_{\mathscr{T}_{q}}\left(x_{k}\right) \in[0,1], \quad(1 / h) \sum_{q=1}^{h}{\Phi_{\delta_{\delta_{q}}}}\left(x_{k}\right) \cdot \quad{\omega_{\delta_{\mathscr{T}_{q}}}}\left(x_{k}\right) \epsilon$ $[0,1],(1 / g) \sum_{p=1}^{g} \gamma_{\mathcal{S}_{p}}^{2}\left(x_{k}\right) \in[0,1],(1 / g) \sum_{q=1}^{g} \delta_{\mathscr{T}_{p}}^{2}\left(x_{k}\right)$ $\epsilon[0,1],(1 / g) \sum_{p=1}^{g} \omega_{\gamma_{\delta_{p}}}^{2}\left(x_{k}\right) \in[0,1],(1 / g) \sum_{p=1}^{g} \omega_{\gamma_{T_{p}}}^{2}$ $\left(x_{k}\right) \in[0,1],(1 / h) \sum_{q=1}^{h} \delta_{\delta_{q}}^{2}\left(x_{k}\right) \in[0,1],(1 / h) \sum_{q=1}^{h} \delta_{\mathscr{T}_{q}}^{2}$ $\left(x_{k}\right) \in[0,1], \quad(1 / h) \sum_{q=1}^{h} \omega_{\delta_{\delta_{q}}}^{2} \quad\left(x_{k}\right) \in[0,1], \quad(1 / h) \sum_{q=1}^{h}$ $\omega_{\delta_{\mathcal{T}_{q}}}^{2}\left(x_{k}\right) \in[0,1]$, and denominator will always remain greater than nominator, then for $k=1$,

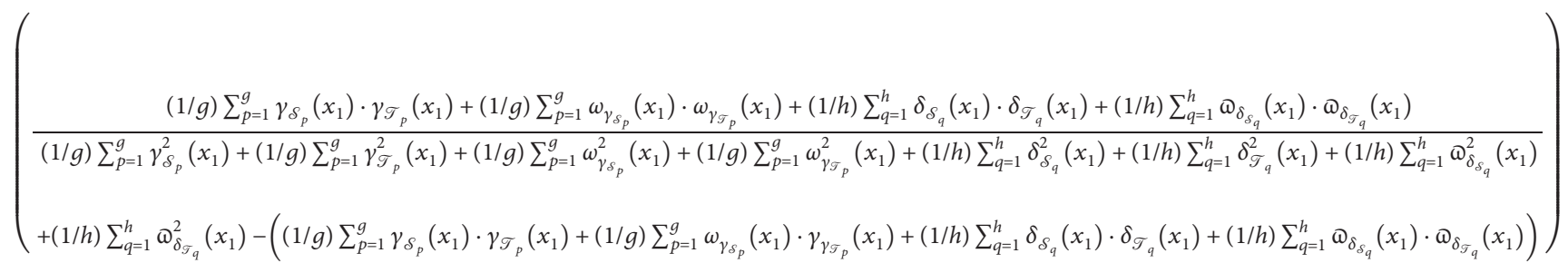

$$
\begin{aligned}
& \in[0,1] .
\end{aligned}
$$

For $k=2$,

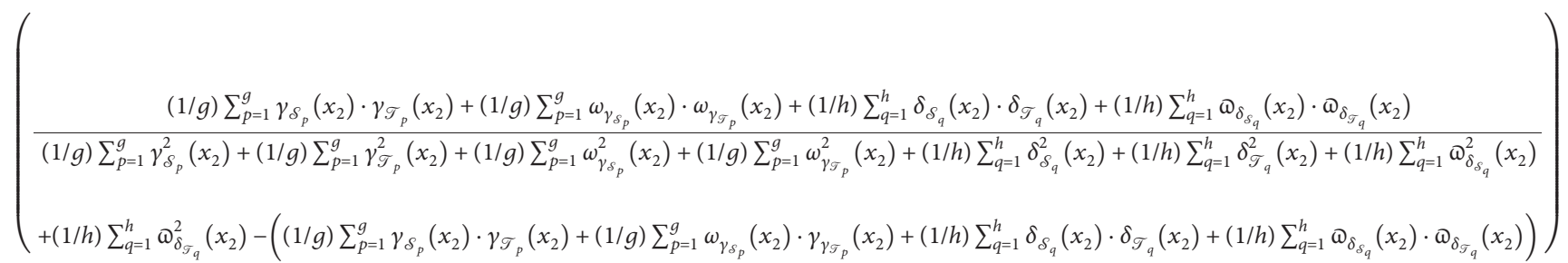

$$
\begin{aligned}
& \epsilon[0,1] .
\end{aligned}
$$

By doing this process, we obtain 


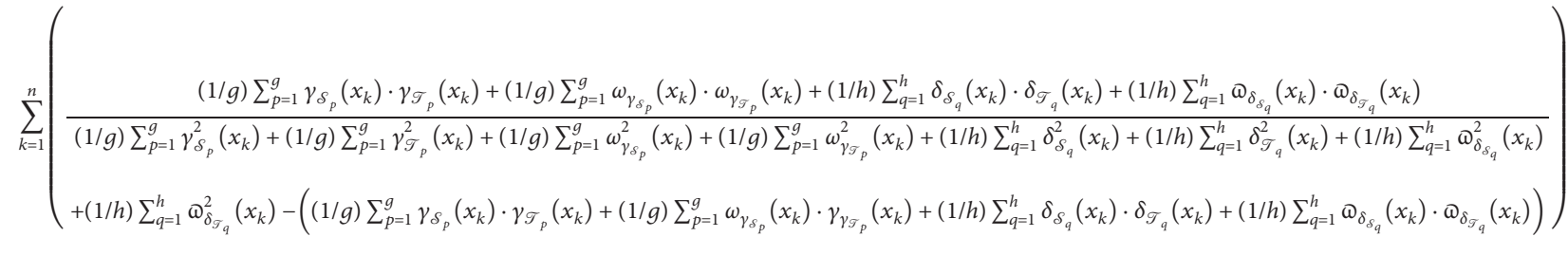

$\in n[0,1]$,

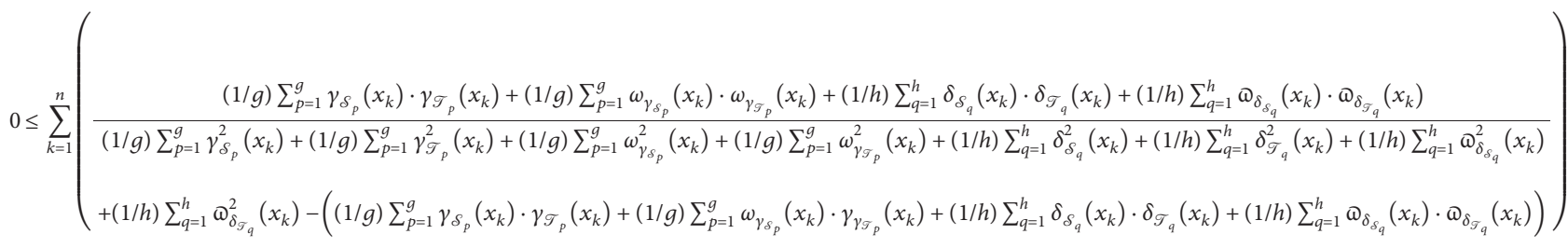

$\leq n$,

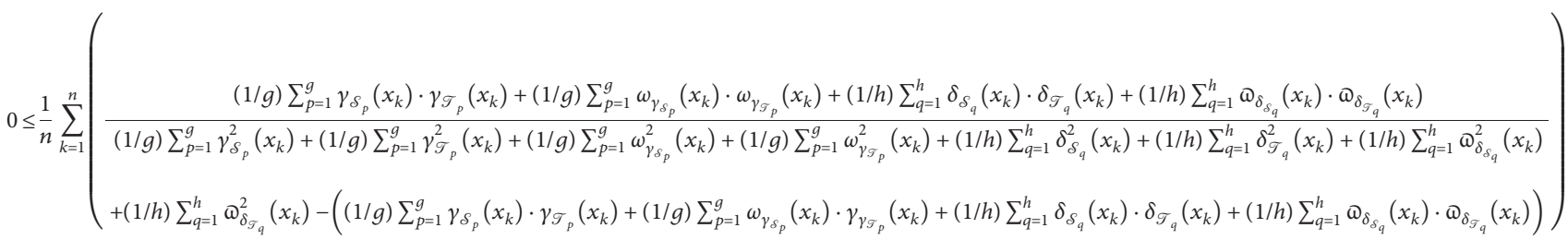

$\leq 1$,

$0 \leq \operatorname{Jac}(\mathcal{S}, \mathscr{T}) \leq 1$.

(2) By definition of JSM, we have

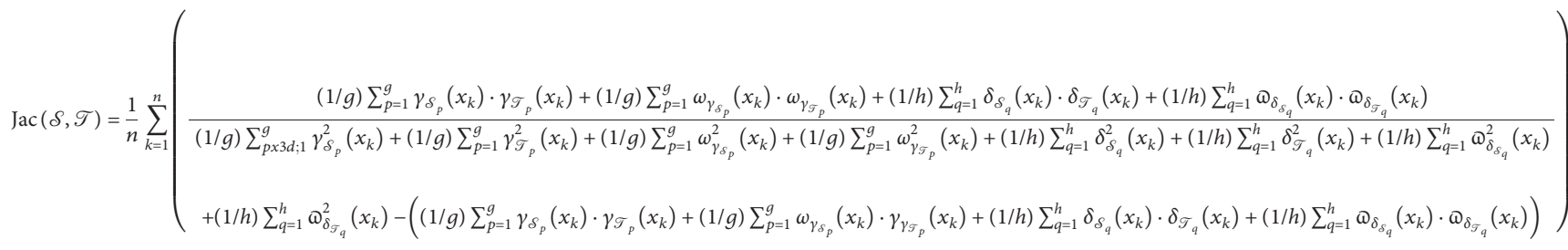

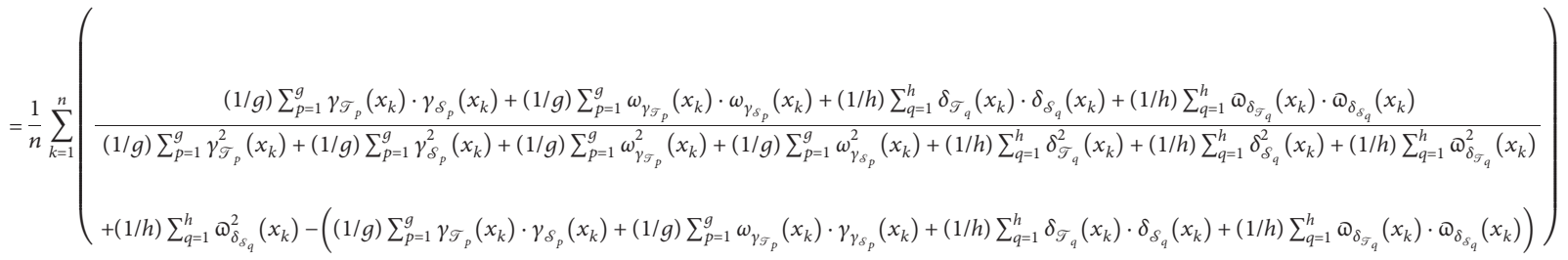$$
=\operatorname{Jac}(\mathscr{T}, \mathcal{S}) .
$$

(3) By definition, we have 


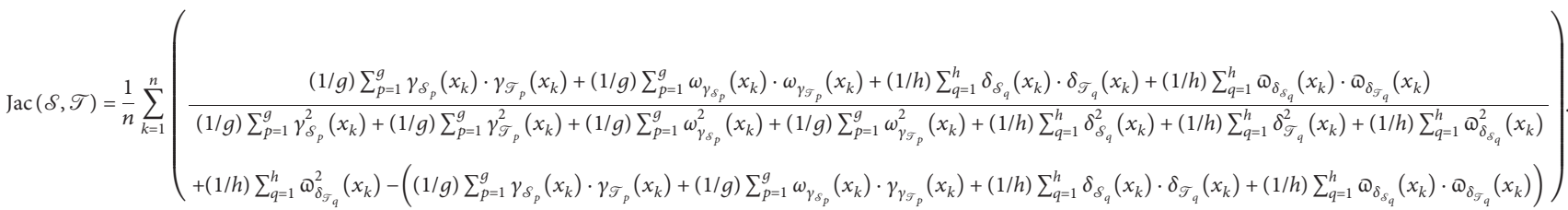

Now as $\quad \mathcal{S}=\mathscr{T} \Longleftrightarrow \mu_{\mathcal{S}}\left(x_{k}\right)=\mu_{\mathscr{T}}\left(x_{k}\right) \quad$ and $v_{\mathcal{S}}\left(x_{k}\right)=v_{\mathscr{T}}\left(x_{k}\right)$ for $k=1,2, \ldots, n \Longleftrightarrow \gamma_{\mathcal{S}_{p}}\left(x_{k}\right) e^{i 2 \pi}\left(\omega_{\gamma_{\mathcal{S}}}\right.$ $\left.\left(x_{k}\right)\right)=\gamma_{\mathscr{T}}\left(x_{k}\right) e^{i 2 \pi}\left(\omega_{\gamma_{\mathscr{T}}}\left(x_{k}\right)\right)$ and $\delta_{\delta_{q}}\left(x_{k}\right) e^{i 2 \pi}\left(\omega_{\gamma_{\delta_{q}}}\right) \stackrel{\gamma_{\delta_{p}}}{=}$ $\left(x_{k}\right), \delta_{\mathcal{S}_{q}}\left(x_{k}\right)=\delta_{\mathscr{T}_{q}}\left(x_{k}\right), e^{i 2 \pi} \quad\left(\omega_{\gamma_{\delta_{p}}}\left(x_{k}\right)\right)=e^{i 2 \pi} \quad\left(\varpi_{\gamma_{T_{p}}}\right.$ $\left.\left(x_{k}\right)\right)$ and $e^{i 2 \pi}\left(\omega_{\delta_{\delta_{q}}}\left(x_{k}^{q}\right)\right)=e^{i 2 \pi}\left(\omega_{\delta_{T_{q}}}\left(x_{k}\right)\right)$, for $k=1,2, \ldots, n$. $\delta_{\mathscr{T}_{q}}\left(x_{k}\right) e^{\eta_{2} \pi}\left(\omega_{\gamma_{\mathscr{T}_{q}}}\right), \quad k \stackrel{p}{=} 1,2, \ldots, n \stackrel{{ }^{2}}{=} \gamma_{\delta_{p}} \quad\left(x_{k}\right) \stackrel{\gamma_{\delta_{q}}}{\gamma_{\mathscr{T}_{p}}}$ Then

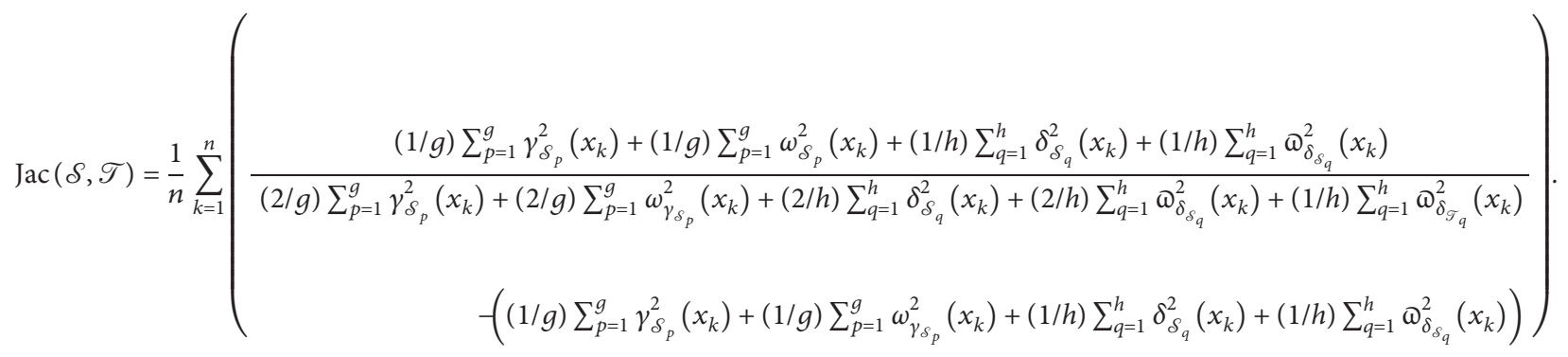

Definition 13. Suppose that $\mathcal{S}$ and $\mathscr{T}$ are two CDHFSs on $X$, then the weighted JSM (WJSM) between $\mathcal{S}$ and $\mathscr{T}$ is denoted and defined as follows:

$\operatorname{Jac}_{w}(\mathcal{S}, \mathscr{T})$

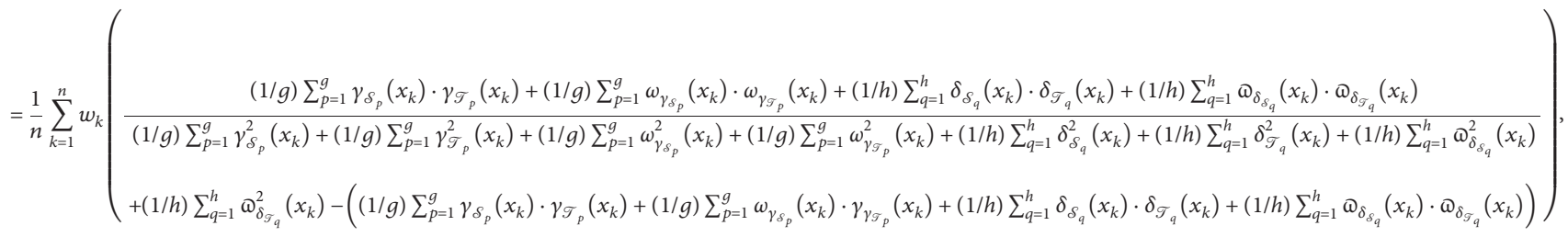

where $w=\left(w_{1}, w_{2}, \ldots, w_{n}\right)^{T}$ speaks to the weight vector of every component $x_{k}(k=1,2,3, \ldots, n)$ contained in CDHFS and the weight vector fulfills $w_{k} \in[0,1]$ for each $k=1,2, \ldots, n, \sum_{k=1}^{n} w_{k}=1$. When we assume the weight vector be $w=((1 / n),(1 / n), \ldots,(1 / n))$, at that point the WJSM will change into JSM. Otherwise, speaking when $w_{k}=(1 / n), k=1,2,3, \ldots, n$, then $\operatorname{Jac}_{w}(\mathcal{S}, \mathscr{T})=$ $\operatorname{Jac}(\mathcal{S}, \mathscr{T})$. 
Definition 14. Suppose that $\mathcal{S}$ and $\mathscr{T}$ are two CDHFSs on $X$, then the Dice similarity measure (DSM) between $\mathcal{S}$ and $\mathscr{T}$ is denoted and defined as follows:

$\operatorname{Dic}(\mathcal{S}, \mathscr{T})$

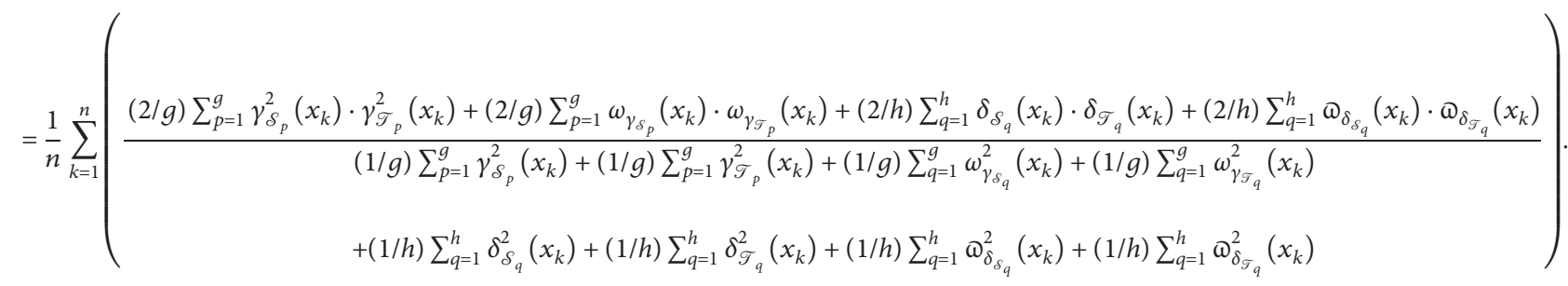

DSMs fulfills the following axioms:

(1) $0 \leq \operatorname{Dic}(\mathcal{S}, \mathscr{T}) \leq 1$

(2) $\operatorname{Dic}(\mathcal{S}, \mathscr{T})=\operatorname{Dic}(\mathscr{T}, \mathcal{S})$

(3) $\operatorname{Dic}(\mathcal{S}, \mathscr{T})=1$, if $\mathcal{S}=\mathscr{T}$

Theorem 2. Prove that equation (17) holds the above three conditions.

Proof.

(1) Since $(1 / g) \sum_{p=1}^{g} \gamma_{\mathcal{S}_{p}}\left(x_{k}\right) \cdot \gamma_{\mathcal{T}_{p}}\left(x_{k}\right) \in[0,1],(1 / g) \sum_{p=1}^{g}$

$$
\gamma_{\delta_{p}}\left(x_{k}\right) \cdot \gamma_{\gamma_{T_{p}}}\left(x_{k}\right) \in[0,1], \quad(1 / h) \sum_{q=1}^{h} \delta_{\delta_{q}}\left(x_{k}\right) \text {. }
$$

$\left(\frac{(2 / g) \sum_{p=1}^{g} \gamma_{\delta_{p}}^{2}\left(x_{1}\right) \cdot \gamma_{\mathscr{T}_{p}}^{2}\left(x_{1}\right)+(2 / g) \sum_{p=1}^{g} \omega_{\gamma_{\delta_{p}}}\left(x_{1}\right) \cdot \omega_{\gamma_{S_{p}}}\left(x_{1}\right)+(2 / h) \sum_{q=1}^{h} \delta_{\delta_{q}}\left(x_{1}\right) \cdot \delta_{\widetilde{T}_{q}}\left(x_{1}\right)+(2 / h) \sum_{q=1}^{h} \omega_{\delta_{\delta_{q}}}\left(x_{1}\right) \cdot \omega_{\delta_{S_{q}}}\left(x_{1}\right)}{(1 / g) \sum_{p=1}^{g} \gamma_{\delta_{p}}^{2}\left(x_{1}\right)+(1 / g) \sum_{p=1}^{g} \gamma_{\mathscr{T}_{p}}^{2}\left(x_{1}\right)+(1 / g) \sum_{q=1}^{g} \omega_{\gamma_{\delta_{q}}}^{2}\left(x_{1}\right)+(1 / g) \sum_{q=1}^{g} \omega_{\gamma_{S_{q}}}^{2}\left(x_{1}\right)+(1 / h) \sum_{q=1}^{h} \delta_{\delta_{q}}^{2}\left(x_{1}\right)+(1 / h) \sum_{q=1}^{h} \delta_{\widetilde{T}_{q}}^{2}\left(x_{1}\right)+(1 / h) \sum_{q=1}^{h} \omega_{\delta_{\delta_{q}}}^{2}\left(x_{1}\right)+(1 / h) \sum_{q=1}^{h} \omega_{\delta_{S_{q}}}^{2}\left(x_{1}\right)}\right)$ $\in n[0,1]$.

For $k=2$, $\epsilon[0,1]$.
$\delta_{\mathscr{T}_{q}}\left(x_{k}\right) \in[0,1],(1 / h) \sum_{q=1}^{h} \omega_{\delta_{\delta_{q}}}\left(x_{k}\right) \cdot \varpi_{\delta_{\mathscr{T}_{q}}}\left(x_{k}\right) \in[0,1]$, $(1 / g) \sum_{p=1}^{g} \gamma_{\mathcal{S}_{p}}^{2}\left(x_{k}\right) \in[0,1],(1 / g) \sum_{q=1}^{g} \delta_{\mathscr{T}_{p}}^{2}\left(x_{k}\right) \in[0,1]$, $(1 / g) \sum_{p=1}^{g} \omega_{\gamma_{\delta_{p}}}^{2}\left(x_{k}\right) \in[0,1], \quad(1 / g) \sum_{p=1}^{g} \quad \omega_{\gamma_{\mathcal{T}_{p}}}^{2}\left(x_{k}\right) \epsilon$ $[0,1],(1 / h) \sum_{q=1}^{h} \delta_{\delta_{q}}^{2}\left(x_{k}\right) \in[0,1],(1 / h) \sum_{q=1}^{h} \delta_{\mathscr{T}_{q}}^{2}\left(x_{k}\right) \epsilon$ $[0,1], \quad(1 / h) \sum_{q=1}^{h} \omega_{\delta_{\delta_{q}}}^{2}\left(x_{k}\right) \in[0,1], \quad(1 / h) \sum_{q=1}^{h} \omega_{\delta_{\mathscr{T}_{q}}}^{2}$ $\left(x_{k}\right) \in[0,1]$, and denominator will always remain greater than nominator, then for $k=1$,

\footnotetext{
$\left(\frac{(2 / g) \sum_{p=1}^{g} \gamma_{\delta_{p}}\left(x_{1}\right) \cdot \gamma_{\widetilde{T}_{p}}\left(x_{2}\right)+(2 / g) \sum_{p=1}^{g} \omega_{\gamma_{p}}\left(x_{2}\right) \cdot \omega_{\gamma_{S_{p}}}\left(x_{2}\right)+(2 / h) \sum_{q=1}^{h} \delta_{\delta_{q}}\left(x_{2}\right) \cdot \delta_{\widetilde{T}_{q}}\left(x_{2}\right)+(2 / h) \sum_{q=1}^{h} \Phi_{\delta_{\delta_{q}}}\left(x_{2}\right) \cdot \omega_{\delta_{S_{q}}}\left(x_{2}\right)}{(1 / g) \sum_{p=1}^{g} \gamma_{\delta_{p}}^{2}\left(x_{2}\right)+(1 / g) \sum_{p=1}^{g} \gamma_{\mathscr{T}_{p}}^{2}\left(x_{2}\right)+(1 / g) \sum_{q=1}^{g} \omega_{\gamma_{\delta_{q}}}^{2}\left(x_{2}\right)+(1 / g) \sum_{q=1}^{g} \omega_{\gamma_{S_{q}}}^{2}\left(x_{2}\right)+(1 / h) \sum_{q=1}^{h} \delta_{\delta_{q}}^{2}\left(x_{2}\right)+(1 / h) \sum_{q=1}^{h} \delta_{\widetilde{T}_{q}}^{2}\left(x_{2}\right)+(1 / h) \sum_{q=1}^{h} \omega_{\delta_{\delta_{q}}}^{2}\left(x_{2}\right)+(1 / h) \sum_{q=1}^{h} \omega_{\delta_{S_{q}}}^{2}\left(x_{2}\right)}\right)$
} 
By doing this process, we obtain

$$
\begin{aligned}
& 0 \leq \sum_{k=1}^{n}\left(\frac{(2 / g) \sum_{p=1}^{g} \gamma_{\delta_{p}}\left(x_{k}\right) \cdot \gamma_{\mathscr{T}_{p}}\left(x_{k}\right)+(2 / g) \sum_{p=1}^{g} \omega_{\gamma_{\delta_{p}}}\left(x_{k}\right) \cdot \omega_{\gamma_{J_{p}}}\left(x_{k}\right)+(2 / h) \sum_{q=1}^{h} \delta_{\delta_{q}}\left(x_{k}\right) \cdot \delta_{\mathscr{T}_{q}}\left(x_{k}\right)+(2 / h) \sum_{q=1}^{h} \Phi_{\delta_{\delta_{q}}}\left(x_{k}\right) \cdot \omega_{\delta_{\delta_{q}}}\left(x_{k}\right)}{(1 / g) \sum_{p=1}^{g} \gamma_{\delta_{p}}^{2}\left(x_{k}\right)+(1 / g) \sum_{p=1}^{g} \gamma_{\mathscr{T}_{p}}^{2}\left(x_{k}\right)+(1 / g) \sum_{q=1}^{g} \omega_{\gamma_{q}}^{2}\left(x_{k}\right)+(1 / g) \sum_{q=1}^{g} \omega_{\gamma_{S_{q}}}^{2}\left(x_{k}\right)+(1 / h) \sum_{q=1}^{h} \delta_{\delta_{q}}^{2}\left(x_{k}\right)+(1 / h) \sum_{q=1}^{h} \delta_{\mathscr{T}_{q}}^{2}\left(x_{k}\right)+(1 / h) \sum_{q=1}^{h} \omega_{\delta_{\delta_{q}}}^{2}\left(x_{k}\right)+(1 / h) \sum_{q=1}^{h} \omega_{\delta_{\sigma_{q}}}^{2}\left(x_{k}\right)}\right) \leq n,
\end{aligned}
$$

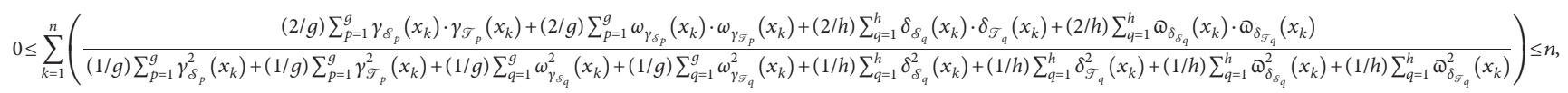

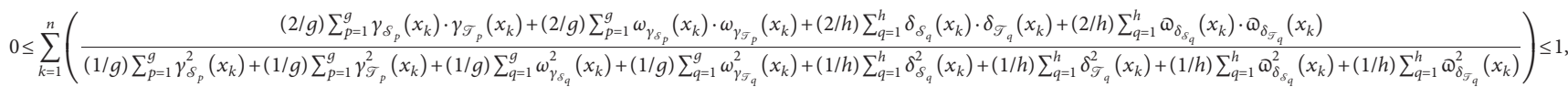

(2) By definition of DSM, we have

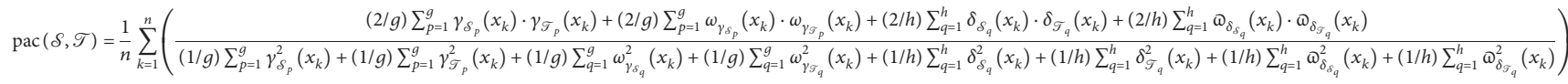

$$
\begin{aligned}
& =\frac{1}{n} \sum_{k=1}^{n} \operatorname{pac}(\mathcal{S}, \mathscr{T}) \\
& =\frac{1}{n} \sum_{k=1}^{n}\left(\frac{(2 / g) \sum_{p=1}^{g} \gamma_{\mathcal{T}_{p}}\left(x_{1}\right) \cdot \gamma_{\delta_{p}}\left(x_{k}\right)+(2 / g) \sum_{p=1}^{g} \omega_{\gamma_{S_{p}}}\left(x_{k}\right) \cdot \omega_{\gamma_{\delta_{p}}}\left(x_{k}\right)+(2 / h) \sum_{q=1}^{h} \delta_{\mathcal{T}_{q}}\left(x_{k}\right) \cdot \delta_{\mathcal{S}_{q}}\left(x_{k}\right)+(2 / h) \sum_{q=1}^{h} \omega_{\delta_{S_{q}}}\left(x_{k}\right) \cdot \Theta_{\delta_{\delta_{q}}}\left(x_{k}\right)}{(1 / g) \sum_{p=1}^{g} \gamma_{\mathcal{F}_{p}}^{2}\left(x_{k}\right)+(1 / g) \sum_{p=1}^{g} \gamma_{\delta_{p}}^{2}\left(x_{k}\right)+(1 / g) \sum_{q=1}^{g} \omega_{\gamma_{S_{q}}}^{2}\left(x_{k}\right)+(1 / g) \sum_{q=1}^{g} \omega_{\gamma_{S_{q}}}^{2}\left(x_{k}\right)+(1 / h) \sum_{q=1}^{h} \delta_{\widetilde{T}_{q}}^{2}\left(x_{k}\right)+(1 / h) \sum_{q=1}^{h} \delta_{\delta_{q}}^{2}\left(x_{k}\right)+(1 / h) \sum_{q=1}^{h} \omega_{\delta_{\delta_{q}}}^{2}\left(x_{k}\right)+(1 / h) \sum_{q=1}^{h} \omega_{\delta_{\delta_{q}}}^{2}\left(x_{k}\right)}\right) \\
& =\operatorname{Dic}(\mathscr{T}, \mathcal{S}) \text {. }
\end{aligned}
$$

(3) By definition, we have

$\operatorname{Dic}(\mathcal{S}, \mathscr{T})$

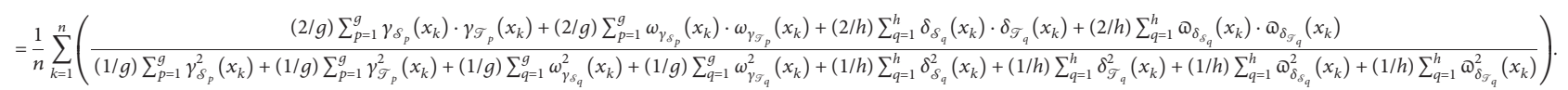

Now as $\mathcal{S}=\mathscr{T} \Longleftrightarrow \mu_{\mathcal{S}}\left(x_{k}\right)=\mu_{\mathscr{T}}\left(x_{k}\right)$ and $v_{\mathcal{S}}\left(x_{k}\right)=$ $v_{\mathscr{T}}\left(x_{k}\right)$ for $k=1,2, \ldots, n \Longleftrightarrow \gamma_{\mathcal{S}_{p}}\left(x_{k}\right) e^{i 2 \pi}\left(\omega_{\gamma_{\delta_{p}}}\left(x_{k}\right)\right)=\gamma_{\mathscr{T}}$ $\left(x_{k}\right) e^{i 2 \pi}\left(\omega_{\gamma_{T_{p}}}\left(x_{k}\right)\right)$ and $\delta_{\delta_{q}}\left(x_{k}\right) e^{i 2 \pi}\left(\omega_{\gamma_{\delta_{q}}}\right) \stackrel{\gamma_{\delta_{p}}}{=} \delta_{\mathscr{T}_{q}}\left(x_{k}\right) e^{i 2 \pi}$
$\left(\oplus_{\gamma_{\overparen{T}}}\right), k=1,2, \ldots, n \Longleftrightarrow \gamma_{\mathcal{S}_{p}}\left(x_{k}\right)=\gamma_{\mathcal{T}_{p}}\left(x_{k}\right), \delta_{\mathcal{S}} \quad\left(x_{k}\right)=$ $\delta_{\mathscr{T}_{q}}\left(x_{k}\right), e^{i 2 \pi}\left(\omega_{\gamma_{\delta_{p}}}\left(x_{k}\right)\right)=e^{i 2 \pi}\left(\Phi_{\gamma_{T_{p}}}\left(x_{k}\right)\right)$ and $e^{i 2 \pi}\left(\omega_{\delta_{\delta_{q}}}\right.$ $\left.\left(x_{k}^{q}\right)\right)=e^{i 2 \pi}\left(\omega_{\delta_{\mathscr{T}_{q}}}\left(x_{k}\right)\right)$ for $k=1,2, \ldots, n$, then

$$
\operatorname{Dic}(\mathcal{S}, \mathscr{T})=\frac{1}{n} \sum_{k=1}^{n}\left(\frac{(2 / g) \sum_{p=1}^{g} \gamma_{\delta_{p}}^{2}\left(x_{k}\right)+(2 / g) \sum_{p=1}^{g} \omega_{\delta_{p}}^{2}\left(x_{k}\right)+(2 / h) \sum_{q=1}^{h} \delta_{\delta_{q}}^{2}\left(x_{k}\right)+(2 / h) \sum_{q=1}^{h} \omega_{\delta_{\delta_{q}}}^{2}\left(x_{k}\right)}{(2 / g) \sum_{p=1}^{g} \gamma_{\delta_{p}}^{2}\left(x_{k}\right)+(2 / g) \sum_{p=1}^{g} \omega_{\delta_{p}}^{2}\left(x_{k}\right)+(2 / h) \sum_{q=1}^{h} \delta_{\delta_{q}}^{2}\left(x_{k}\right)+(2 / h) \sum_{q=1}^{h} \omega_{\delta_{\delta_{q}}}^{2}\left(x_{k}\right)}\right)=1 .
$$


Definition 15. Suppose that $\mathcal{S}$ and $\mathscr{T}$ are two CDHFSs on $X$, then the weighted DSM (WJSM) between $\mathcal{S}$ and $\mathscr{T}$ is denoted and defined as follows:

$$
\begin{aligned}
& \operatorname{Dic}(\mathcal{S}, \mathscr{T}) \\
& =\sum_{k=1}^{n} w_{k}\left(\frac{(2 / g) \sum_{p=1}^{g} \gamma_{\mathcal{S}_{p}}\left(x_{k}\right) \cdot \gamma_{\mathscr{T}_{p}}\left(x_{k}\right)+(2 / g) \sum_{p=1}^{g} \omega_{\gamma_{S_{p}}}\left(x_{k}\right) \cdot \omega_{\gamma_{S_{p}}}\left(x_{k}\right)+(2 / h) \sum_{q=1}^{h} \delta_{\delta_{q}}\left(x_{k}\right) \cdot \delta_{\widetilde{T}_{q}}\left(x_{k}\right)+(2 / h) \sum_{q=1}^{h} \omega_{\delta_{\delta_{q}}}\left(x_{k}\right) \cdot \omega_{\delta_{\mathcal{S}_{q}}}\left(x_{k}\right)}{(1 / g) \sum_{p=1}^{g} \gamma_{\delta_{p}}^{2}\left(x_{k}\right)+(1 / g) \sum_{p=1}^{g} \gamma_{\mathscr{T}_{p}}^{2}\left(x_{k}\right)+(1 / g) \sum_{q=1}^{g} \omega_{\gamma_{S_{q}}}^{2}\left(x_{k}\right)+(1 / g) \sum_{q=1}^{g} \omega_{\gamma_{S_{q}}}^{2}\left(x_{k}\right)+(1 / h) \sum_{q=1}^{h} \delta_{\delta_{q}}^{2}\left(x_{k}\right)+(1 / h) \sum_{q=1}^{h} \delta_{\mathscr{T}_{q}}^{2}\left(x_{k}\right)+(1 / h) \sum_{q=1}^{h} \omega_{\delta_{\delta_{q}}}^{2}\left(x_{k}\right)+(1 / h) \sum_{q=1}^{h} \omega_{\delta_{S_{q}}}^{2}\left(x_{k}\right)}\right),
\end{aligned}
$$

where $w=\left(w_{1}, w_{2}, \ldots, w_{n}\right)^{T}$ speaks to the weight vector of every component $x_{k}(k=1,2,3, \ldots, n)$ contained in CDHFS and the weight vector fulfills $w_{k} \in[0,1]$ for each $k=1,2,3, \ldots, n, \sum_{k=1}^{n} w_{k}=1$. When we assume the weight vector be $w=((1 / n),(1 / n), \ldots,(1 / n))^{T}$, at that point the WDSM will change into DSM. Otherwise, speaking when $w_{k}=(1 / n), k=1,2,3, \ldots, n$,

then

$\operatorname{Dic}_{w}(\mathcal{S}, \mathscr{T})=\operatorname{Dic}(\mathcal{S}, \mathscr{T})$.

Definition 16. Suppose that $\delta$ and $\mathscr{T}$ are two CDHFSs on $X$, then the Cosine similarity measure (CSM) between $\mathcal{S}$ and $\mathscr{T}$ is denoted and defined as follows:

$\operatorname{Cos}(\mathcal{S}, \mathscr{T})$

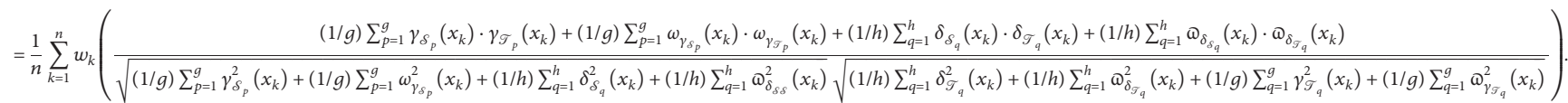

CSMs fulfill the following axioms:

(1) $0 \leq \operatorname{Cos}(\mathcal{S}, \mathscr{T}) \leq 1$

(2) $\operatorname{Cos}(\mathcal{S}, \mathscr{T})=\operatorname{Cos}(\mathscr{T}, \mathcal{S})$

(3) $\operatorname{Cos}(\mathcal{S}, \mathscr{T})=1$, if $\mathcal{S}=\mathscr{T}$

Theorem 3. Prove that equation (25), holds the above three conditions.

Proof.

(1) Since $\quad(1 / g) \sum_{p=1}^{g} \gamma_{\mathcal{S}_{p}}\left(x_{k}\right) \cdot \gamma_{\mathscr{T}_{p}}\left(x_{k}\right) \in[0,1], \quad(1 / g)$ $\sum_{p=1}^{g} \omega_{\gamma_{\delta_{p}}}\left(x_{k}\right) \cdot \omega_{\gamma_{\widetilde{T}_{p}}}\left(x_{k}\right) \in[0,1],(1 / h) \sum_{q=1}^{h} \delta_{\delta_{q}}\left(x_{k}\right)$.
$\delta_{\mathscr{T}_{q}}\left(x_{k}\right) \in[0,1],(1 / h) \sum_{q=1}^{h} \omega_{\delta_{\delta_{q}}}\left(x_{k}\right) \cdot{\omega_{\delta_{\mathcal{T}_{q}}}}\left(x_{k}\right) \in[0,1]$, $(1 / g) \sum_{p=1}^{g} \gamma_{\mathcal{S}_{p}}^{2}\left(x_{k}\right) \in[0,1], \quad(1 / g) \sum_{q=1}^{g} \delta_{\mathscr{T}_{p}}^{2}\left(x_{k}\right) \epsilon$ $[0,1], \quad(1 / g) \sum_{p=1}^{g} \omega_{\gamma_{\delta_{p}}}^{2}\left(x_{k}\right) \in[0,1], \quad(1 / g) \sum_{p=1}^{g} \omega_{\gamma_{\mathscr{T}}}^{2}$ $\left(x_{k}\right) \in[0,1],(1 / h) \sum_{q=1}^{h} \delta_{\delta_{q}}^{2}\left(x_{k}\right) \in[0,1],(1 / h) \sum_{q=1}^{h} \delta_{\mathscr{T}_{q}}^{2}$ $\left(x_{k}\right) \in[0,1], \quad(1 / h) \sum_{q=1}^{h} \quad \Phi_{\delta_{\delta_{q}}}^{2}\left(x_{k}\right) \in[0,1]$, $(1 / h) \sum_{q=1}^{h} \omega_{\delta_{T_{q}}}^{2}\left(x_{k}\right) \in[0,1]$, and denominator will always remain greater than nominator, then for $k=1$,

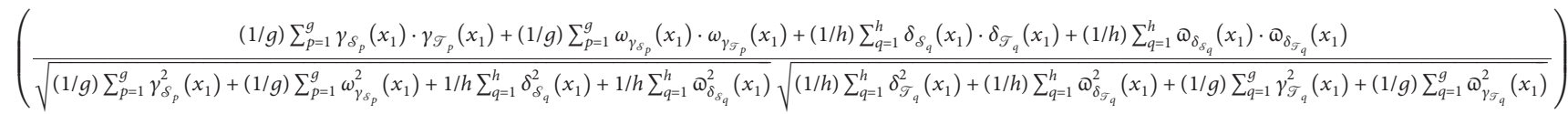
$\in[0,1]$. 
For $k=2$,

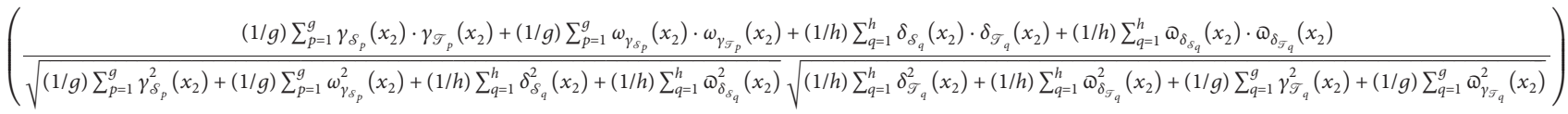
$\in[0,1]$.

By doing this process, we obtain

$$
\begin{aligned}
& \sum_{k=1}^{n}\left(\frac{(1 / g) \sum_{p=1}^{g} \gamma_{\delta_{p}}\left(x_{k}\right) \cdot \gamma_{\widetilde{S}_{p}}\left(x_{k}\right)+(1 / g) \sum_{p=1}^{g} \omega_{\gamma_{p}}\left(x_{k}\right) \cdot \omega_{\gamma_{S_{p}}}\left(x_{k}\right)+(1 / h) \sum_{q=1}^{h} \delta_{\delta_{q}}\left(x_{2 k}\right) \cdot \delta_{\widetilde{T}_{q}}\left(x_{k}\right)+(1 / h) \sum_{q=1}^{h} \omega_{\delta_{\delta_{q}}}\left(x_{k}\right) \cdot \omega_{\delta_{\delta_{q}}}\left(x_{k}\right)}{\sqrt{(1 / g) \sum_{p=1}^{g} \gamma_{\delta_{p}}^{2}\left(x_{k}\right)+(1 / g) \sum_{p=1}^{g} \omega_{\gamma_{S_{p}}}^{2}\left(x_{k}\right)+(1 / h) \sum_{q=1}^{h} \delta_{\delta_{q}}^{2}\left(x_{k}\right)+(1 / h) \sum_{q=1}^{h} \omega_{\delta_{\delta_{q}}}^{2}\left(x_{k}\right)} \sqrt{(1 / h) \sum_{q=1}^{h} \delta_{\mathscr{S}_{q}}^{2}\left(x_{k}\right)+(1 / h) \sum_{q=1}^{h} \omega_{\delta_{S_{q}}}^{2}\left(x_{k}\right)+(1 / g) \sum_{q=1}^{g} \gamma_{\mathscr{S}_{q}}^{2}\left(x_{k}\right)+(1 / g) \sum_{q=1}^{g} \omega_{\gamma_{S_{q}}}^{2}\left(x_{k}\right)}}\right) \epsilon n[0,1], \\
& 0 \leq \sum_{k=1}^{n}\left(\frac{(1 / g) \sum_{p=1}^{g} \gamma_{\delta_{p}}\left(x_{k}\right) \cdot \gamma_{\mathcal{S}_{p}}\left(x_{k}\right)+(1 / g) \sum_{p=1}^{g} \omega_{\gamma_{\delta_{p}}}\left(x_{k}\right) \cdot \omega_{\gamma_{S_{p}}}\left(x_{k}\right)+(1 / h) \sum_{q=1}^{h} \delta_{\delta_{q}}\left(x_{2 k}\right) \cdot \delta_{\widetilde{J}_{q}}\left(x_{k}\right)+(1 / h) \sum_{q=1}^{h} \omega_{\delta_{\delta_{q}}}\left(x_{k}\right) \cdot \omega_{\delta_{\delta_{q}}}\left(x_{k}\right)}{\sqrt{(1 / g) \sum_{p=1}^{g} \gamma_{\delta_{p}}^{2}\left(x_{k}\right)+(1 / g) \sum_{p=1}^{g} \omega_{\gamma_{\delta_{p}}}^{2}\left(x_{k}\right)+(1 / h) \sum_{q=1}^{h} \delta_{\delta_{q}}^{2}\left(x_{k}\right)+(1 / h) \sum_{q=1}^{h} \omega_{\delta_{\delta_{q}}}^{2}\left(x_{k}\right)} \sqrt{(1 / h) \sum_{q=1}^{h} \delta_{\widetilde{S}_{q}}^{2}\left(x_{k}\right)+(1 / h) \sum_{q=1}^{h} \omega_{\delta_{\delta_{q}}}^{2}\left(x_{k}\right)+(1 / g) \sum_{q=1}^{g} \gamma_{\widetilde{S}_{q}}^{2}\left(x_{k}\right)+(1 / g) \sum_{q=1}^{g} \omega_{\gamma_{S_{q}}}^{2}\left(x_{k}\right)}}\right) \leq n,
\end{aligned}
$$

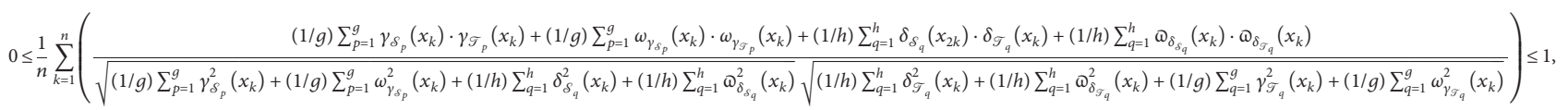

$$
\begin{aligned}
& 0 \leq \operatorname{Cos}(\mathcal{S}, \mathscr{T}) \leq 1 .
\end{aligned}
$$

(2) By definition of CSM, we have

$\operatorname{Cos}(\mathcal{S}, \mathscr{T})$

$=\frac{1}{n} \sum_{k=1}^{n}\left(\frac{(1 / g) \sum_{p=1}^{g} \gamma_{\delta_{p}}\left(x_{k}\right) \cdot \gamma_{\mathscr{T}_{p}}\left(x_{k}\right)+(1 / g) \sum_{p=1}^{g} \omega_{\gamma_{\delta_{p}}}\left(x_{k}\right) \cdot \omega_{\gamma_{T_{p}}}\left(x_{k}\right)+(1 / h) \sum_{q=1}^{h} \delta_{\delta_{q}}\left(x_{2 k}\right) \cdot \delta_{\mathscr{T}_{q}}\left(x_{k}\right)+(1 / h) \sum_{q=1}^{h} \omega_{\delta_{\delta_{q}}}\left(x_{k}\right) \cdot \omega_{\delta_{T_{q}}}\left(x_{k}\right)}{\sqrt{(1 / g) \sum_{p=1}^{g} \gamma_{\delta_{p}}^{2}\left(x_{k}\right)+(1 / g) \sum_{p=1}^{g} \omega_{\gamma_{\delta_{p}}}^{2}\left(x_{k}\right)+(1 / h) \sum_{q=1}^{h} \delta_{\delta_{q}}^{2}\left(x_{k}\right)+(1 / h) \sum_{q=1}^{h} \omega_{\delta_{\delta_{q}}}^{2}\left(x_{k}\right)} \sqrt{(1 / h) \sum_{q=1}^{h} \delta_{\mathscr{T}_{q}}^{2}\left(x_{k}\right)+(1 / h) \sum_{q=1}^{h} \omega_{\delta_{\delta_{q}}}^{2}\left(x_{k}\right)+(1 / g) \sum_{q=1}^{g} \gamma_{\mathscr{T}_{q}}^{2}\left(x_{k}\right)+(1 / g) \sum_{q=1}^{g} \omega_{\gamma_{S_{q}}}^{2}\left(x_{k}\right)}}\right)$ $=\operatorname{Cos}(\mathscr{T}, \mathcal{S})$.

(3) By definition, we have

$\operatorname{Cos}(\mathcal{S}, \mathscr{T})$

$=\frac{1}{n} \sum_{k=1}^{n}\left(\frac{(1 / g) \sum_{p=1}^{g} \gamma_{\delta_{p}}\left(x_{k}\right) \cdot \gamma_{\mathscr{T}_{p}}\left(x_{k}\right)+(1 / g) \sum_{p=1}^{g} \omega_{\gamma_{\delta_{p}}}\left(x_{k}\right) \cdot \omega_{\gamma_{J_{p}}}\left(x_{k}\right)+(1 / h) \sum_{q=1}^{h} \delta_{\delta_{q}}\left(x_{2 k}\right) \cdot \delta_{\mathscr{T}_{q}}\left(x_{k}\right)+(1 / h) \sum_{q=1}^{h} \omega_{\delta_{\delta_{q}}}\left(x_{k}\right), \ldots,\left(x_{k}\right)}{\sqrt{(1 / g) \sum_{p=1}^{g} \gamma_{\delta_{p}}^{2}\left(x_{k}\right)+(1 / g) \sum_{p=1}^{g} \omega_{\gamma_{S_{p}}}^{2}\left(x_{k}\right)+(1 / h) \sum_{q=1}^{h} \delta_{\delta_{q}}^{2}\left(x_{k}\right)+(1 / h) \sum_{q=1}^{h} \omega_{\delta_{\delta_{q}}}^{2}\left(x_{k}\right)} \sqrt{(1 / h) \sum_{q=1}^{h} \delta_{\mathscr{T}_{q}}^{2}\left(x_{k}\right)+(1 / h) \sum_{q=1}^{h} \omega_{\delta_{S_{q}}}^{2}\left(x_{k}\right)+(1 / g) \sum_{q=1}^{g} \gamma_{\mathscr{T}_{q}}^{2}\left(x_{k}\right)+(1 / g) \sum_{q=1}^{g} \omega_{\gamma_{S_{q}}}^{2}\left(x_{k}\right)}}\right)$.

Now as $\mathcal{S}=\mathscr{T} \Longleftrightarrow \mu_{\mathcal{S}}\left(x_{k}\right)=\mu_{\mathscr{T}}\left(x_{k}\right)$ and $v_{\mathcal{S}}\left(x_{k}\right)=$ $v_{\mathscr{T}}\left(x_{k}\right)$ for $k=1,2, \ldots, n \Longleftrightarrow \gamma_{\mathcal{S}_{p}}\left(x_{k}\right) e^{i 2 \pi}\left(\omega_{\gamma_{\delta_{p}}}\left(x_{k}\right)\right)=\gamma_{\mathscr{T}_{p}}$ $\left(x_{k}\right) e^{i 2 \pi}\left(\omega_{\gamma_{T_{p}}}\left(x_{k}\right)\right)$ and $\delta_{\mathcal{S}_{q}}\left(x_{k}\right) e^{i 2 \pi}\left({\Phi_{\gamma_{\delta_{q}}}}\right)=\delta_{\mathscr{T}_{q}}\left(x_{k}\right) e^{i 2 \pi}$
$\left(\Phi_{\gamma_{T_{q}}}\right), k=1,2, \ldots, n \Longleftrightarrow \gamma_{\mathcal{S}_{p}}\left(x_{k}\right)=\gamma_{\mathscr{T}_{p}}\left(x_{k}\right), \delta_{\delta_{q}}\left(x_{k}\right)=$ $\delta_{\mathscr{T}_{q}}\left(x_{k}\right), e^{i 2 \pi}\left(\omega_{\gamma_{\delta_{p}}}\left(x_{k}\right)\right)=e^{i 2 \pi}\left({\omega_{\gamma_{T_{p}}}}\left(x_{k}\right)\right)$ and $e^{i 2 \pi}\left({\Phi_{\delta_{\delta_{q}}}}\right.$ $\left.\left(x_{k}\right)\right)=e^{i 2 \pi}\left({\Phi_{\delta_{\mathscr{T}_{q}}}}\left(x_{k}\right)\right)$ for $k=1,2, \ldots, n$, then 


$$
\operatorname{Cos}(\mathcal{S}, \mathscr{T})=\frac{1}{n} \sum_{k=1}^{n}\left(\frac{(1 / g) \sum_{p=1}^{g} \gamma_{\delta_{p}}^{2}\left(x_{k}\right)+(1 / g) \sum_{p=1}^{g} \omega_{\delta_{p}}^{2}\left(x_{k}\right)+(1 / h) \sum_{q=1}^{h} \delta_{\delta_{q}}^{2}\left(x_{2 k}\right)+(1 / h) \sum_{q=1}^{h} \Phi_{\delta_{\delta_{q}}}^{2}\left(x_{k}\right)}{\left(\sqrt{(1 / g) \sum_{p=1}^{g} \gamma_{\delta_{p}}^{2}\left(x_{k}\right)+(1 / g) \sum_{p=1}^{g} \omega_{\gamma_{\delta_{p}}}^{2}\left(x_{k}\right)+(1 / h) \sum_{q=1}^{h} \delta_{\delta_{q}}^{2}\left(x_{k}\right)+(1 / h) \sum_{q=1}^{h} \Phi_{\delta_{\delta_{q}}}^{2}\left(x_{k}\right)}\right)^{2}}\right)=1
$$

Definition 17. Suppose that $\mathcal{S}$ and $\mathscr{T}$ are two CDHFSs on $X$, then the weighted CSM (WCSM) between $\delta$ and $\mathscr{T}$ is denoted and defined as follows:

$\operatorname{Cos}(\mathcal{S}, \mathscr{T})$

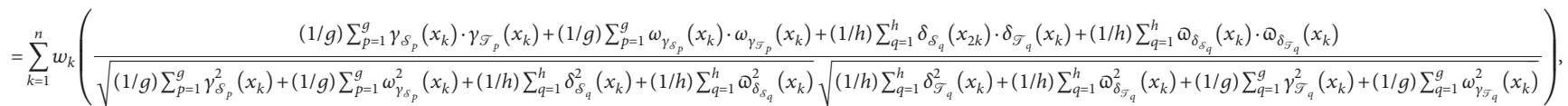

where $w=\left(w_{1}, w_{2}, \ldots, w_{n}\right)^{T}$ speaks to the weight vector of every component $x_{k}(k=1,2,3, \ldots, n)$ contained in CDHFS and the weight vector fulfills $w_{k} \in[0,1]$ for each $k=1,2,3, \ldots, n, \sum_{k=1}^{n} w_{k}=1$. When we assume the weight vector be $w=((1 / n),(1 / n), \ldots,(1 / n))^{T}$, at that point the WCSM will change into CSM. Otherwise, speaking when $w_{k}=(1 / n), k=1,2,3, \ldots, n$, $\operatorname{Dic}_{w}(\mathcal{S}, \mathscr{T})=\operatorname{Dic}(\mathcal{S}, \mathscr{T})$.
4.2. Hybrid Vector Similarity Measure. In this section, we characterize hybrid vector similarity measure and weighted hybrid vector similarity measure for complex dual hesitant fuzzy sets.

Definition 18. Suppose that $\mathcal{S}$ and $\mathscr{T}$ are two CDHFSs on $X$, then the hybrid VSM between $\mathcal{S}$ and $\mathscr{T}$ is denoted and defined as follows:

$\operatorname{Hyb}(\mathcal{S}, \mathscr{T})$

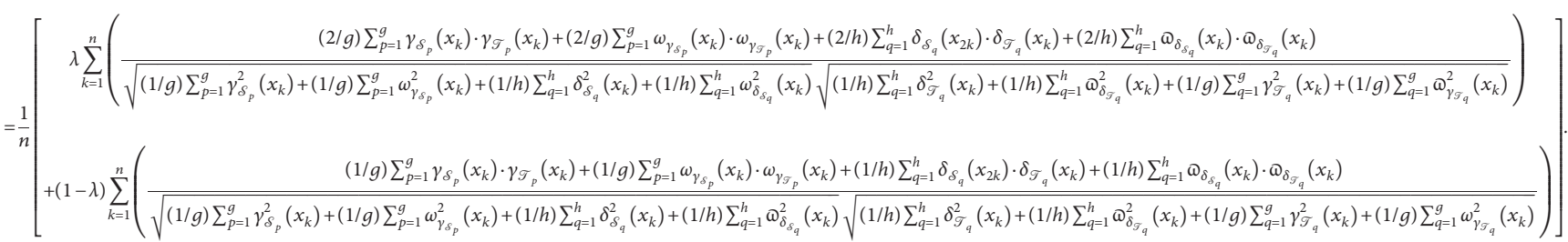

Definition 19. Suppose that $\delta$ and $\mathscr{T}$ are two CDHFSs on $X$, then the weighted hybrid VSM between $\mathcal{S}$ and $\mathscr{T}$ is denoted and defined as follows:

$\operatorname{Hyb}_{w}(\mathcal{S}, \mathscr{T})$

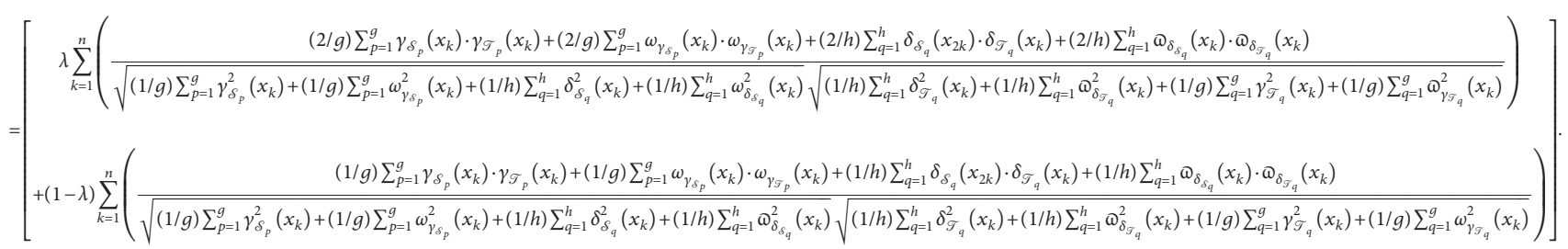


The weighted hybrid VSM of CDHFS fulfill the following axioms:

(1) $0 \leq \operatorname{Hyb}_{w}(\mathcal{S}, \mathscr{T}) \leq 1$

(2) $\operatorname{Hy}(\mathcal{S}, \mathscr{T})=\operatorname{Hyb}_{w}(\mathscr{T}, \mathcal{S})$

(3) $\operatorname{Hyb}_{w}(\mathcal{S}, \mathscr{T})=1$, if $\mathcal{S}=\mathscr{T}$

Theorem 4. Prove that equation (34) holds the above three conditions.

Proof (1) From equations (24) and (32), we have $0 \leq \operatorname{Dic}_{w} \leq 1$ and $0 \leq \operatorname{Cos}_{w} \leq 1$ for all $k=1,2, \ldots, n$. Thus, equation (34) becomes

$$
\begin{aligned}
\operatorname{Hyb}_{w}(\mathcal{S}, \mathscr{T}) & =\lambda \operatorname{Dic}_{w}(\mathcal{S}, \mathscr{T})+(1-\lambda) \operatorname{Cos}_{w}(\mathcal{S}, \mathscr{T}) \\
& \leq \lambda+(1-\lambda)=1,
\end{aligned}
$$

because $\operatorname{Dic}_{w}(\mathcal{S}, \mathscr{T}) \geq 0$ and $\operatorname{Cos}_{w}(\mathcal{S}, \mathscr{T}) \geq 0$; this implies that $\operatorname{Hyb}_{w}(\mathcal{S}, \mathscr{T}) \geq 0$ for any value of $\lambda \in[0,1]$. This completes the prove of 1 .

(2) Since $\operatorname{Dic}_{w}(\mathcal{S}, \mathscr{T})=\operatorname{Dic}_{w}(\mathscr{T}, \mathcal{S}) \quad$ and $\operatorname{Cos}_{w}(\mathcal{S}, \mathscr{T})=\operatorname{Cos}_{w}(\mathscr{T}, \mathcal{S})$, this implies that.

(3) If $\mathcal{S}=\mathscr{T}$, then from equation (24) and (32) we have $\operatorname{Dic}_{w}(\mathcal{S}, \mathscr{T})=1$ and $\operatorname{Cos}_{w}(\mathcal{S}, \mathscr{T})=1$; this implies that $\operatorname{Hyb}_{w}(\mathcal{S}, \mathscr{T})=\lambda+(1-\lambda)=1$.

\section{Applications}

In this section of the article, we portray two applications, for example, pattern recognition and medical diagnosis to show the viability and value of the deciphered SMS. We applied the explored SMs to the environment of CDHFSs in pattern recognition and medical diagnosis.

5.1. Pattern Recognition. The tools of similarity measures have applications in pattern classification. In such a marvel, the class of an obscure pattern or object discovered abuse some closeness estimating instruments and scarcely any inclinations of decision makers. In this section, the similarity measures developed up to this point in Section 4 are applied to a pattern recognition (building pattern recognition) drawback any place the class of an obscure building substance has been evaluated. The results procured using the SMs of CDHFSs are then explored for delineation of the upsides of proposed SMs and the containments of existing work. To explain the wonder, the underneath explained is examined.

Example 3. Its feed that amounts from developments by an organization is legitimately corresponding to the standard of building substances they use. An appropriate review of the building substance before development is the confirmation of good building measures. The building substances to be utilized ought to be carefully checked before applying. The best possible check and equalization arrangement of investigation approve the manufacturers to utilize the correct substances for developments to improve the standard of their task. Five known building substances $\mathcal{S}_{r}(r=1,2,3,4,5)$ which are given in the CDHFSs structure are as follows:

$$
\begin{aligned}
\mathcal{S}_{1}= & \left\{\left(x_{1},\left\{\left\{0.2 e^{i 2 \pi(0.15)}, 0.3 e^{i 2 \pi(0.2)}, 0.5 e^{i 2 \pi(0.4)}\right\},\left\{0.3 e^{i 2 \pi(0.5)}, 0.2 e^{i 2 \pi(0.25)}\right\}\right\}\right),\left(x_{2},\left\{\left\{0.3 e^{i 2 \pi(0.2)}\right\},\left\{0.6 e^{i 2 \pi(0.5)}, 0.4 e^{i 2 \pi(0.35)}\right\}\right\}\right),\right. \\
& \left.\left(x_{3},\left\{\left\{0.6 e^{i 2 \pi(0.1)}\right\},\left\{0.2 e^{i 2 \pi(0.4)}\right\}\right\}\right),\left(x_{4},\left\{\left\{0.15 e^{i 2 \pi(0.25)}, 0.6 e^{i 2 \pi(0.5)}\right\},\left\{0.35 e^{i 2 \pi(0.15)}\right\}\right\}\right),\left(x_{5},\left\{\left\{0.4 e^{i 2 \pi(0.65)}\right\},\left\{0.3 e^{i 2 \pi(0.2)}\right\}\right\}\right)\right\}, \\
\mathcal{S}_{2}= & \left\{\left(x_{1},\left\{\left\{0.25 e^{i 2 \pi(0.1)}\right\},\left\{0.6 e^{i 2 \pi(0.7)}, 0.3 e^{i 2 \pi(0.5)}\right\}\right\}\right),\left(x_{2},\left\{\left\{0.35 e^{i 2 \pi(0.3)}\right\},\left\{0.5 e^{i 2 \pi(0.4)}\right\}\right\}\right),\right. \\
& \left(x_{3},\left\{\left\{0.35 e^{i 2 \pi(0.4)}, 0.45 e^{i 2 \pi(0.5)}, 0.6 e^{i 2 \pi(0.35)}\right\},\left\{0.3 e^{i 2 \pi(0.45)}, 0.1 e^{i 2 \pi(0.25)}\right\}\right\}\right), \\
& \left.\left(x_{4},\left\{\left\{0.6 e^{i 2 \pi(0.33)}, 0.5 e^{i 2 \pi(0.45)}\right\},\left\{0.2 e^{i 2 \pi(0.5)}, 0.25 e^{i 2 \pi(0.3)}\right\}\right\}\right)\left(x_{5},\left\{\left\{0.5 e^{i 2 \pi(0.4)}\right\},\left\{0.25 e^{i 2 \pi(0.45)}, 0.4 e^{i 2 \pi(0.35)}\right\}\right\}\right)\right\}, \\
\mathcal{S}_{3}= & \left\{\left(x_{1},\left\{\left\{0.3 e^{i 2 \pi(0.4)}, 0.2 e^{i 2 \pi(0.45)}\right\},\left\{0.4 e^{i 2 \pi(0.3)}, 0.5 e^{i 2 \pi(0.1)}\right\}\right\}\right),\left(x_{2},\left\{\left\{0.45 e^{i 2 \pi(0.7)}, 0.5 e^{i 2 \pi(0.65)}\right\},\left\{0.15 e^{i 2 \pi(0.2)}\right\}\right\}\right),\right. \\
& \left(x_{3},\left\{\left\{0.6 e^{i 2 \pi(0.5)}\right\},\left\{0.35 e^{i 2 \pi(0.4)}\right\}\right\}\right),\left(x_{4},\left\{\left\{0.7 e^{i 2 \pi(0.5)}\right\},\left\{0.25 e^{i 2 \pi(0.3)}, 0.15 e^{i 2 \pi(0.1)}\right\}\right\}\right), \\
& \left.\left(x_{5},\left\{\left\{0.1 e^{i 2 \pi(0.05)}, 0.25 e^{i 2 \pi(0.15)}, 0.4 e^{i 2 \pi(0.45)}\right\},\left\{0.5 e^{i 2 \pi(0.5)}, 0.2 e^{i 2 \pi(0.3)}\right\}\right\}\right)\right\},
\end{aligned}
$$


TABLE 1: Estimations of the interpreted VSMs.

\begin{tabular}{|c|c|c|c|c|c|c|}
\hline Similarity measures & $\left(\mathcal{S}, \mathcal{S}_{1}\right)$ & $\left(\mathcal{S}, \mathcal{S}_{2}\right)$ & $\left(\mathcal{S}, \mathcal{S}_{3}\right)$ & $\left(\mathcal{S}, \mathcal{S}_{4}\right)$ & $\left(\mathcal{S}, \mathcal{S}_{5}\right)$ & Ranking \\
\hline $\operatorname{Jac}\left(\mathcal{S}, \mathcal{S}_{r}\right)$ & 0.3936 & 0.4455 & 0.2748 & 0.6742 & 0.4087 & $\mathcal{S}_{4} \geq \mathcal{S}_{2} \geq \mathcal{S}_{5} \geq \mathcal{S}_{1} \geq \mathcal{S}_{3}$ \\
\hline $\operatorname{Dic}\left(\mathcal{S}, \mathcal{S}_{r}\right)$ & 0.5353 & 0.581 & 0.4253 & 0.7755 & 0.5504 & $\mathcal{S}_{4} \geq \mathcal{S}_{2} \geq \mathcal{S}_{5} \geq \mathcal{S}_{1} \geq \mathcal{S}_{3}$ \\
\hline $\operatorname{Cos}\left(\mathcal{S}, \mathcal{S}_{r}\right)$ & 0.5465 & 0.6048 & 0.447 & 0.7444 & 0.557 & $\mathcal{S}_{4} \geq \mathcal{S}_{2} \geq \mathcal{S}_{5} \geq \mathcal{S}_{1} \geq \mathcal{S}_{3}$ \\
\hline
\end{tabular}

TABle 2: Estimations of the interpreted WVSMs.

\begin{tabular}{lcccccc}
\hline Similarity measures & $\left(\mathcal{S}, \mathcal{S}_{1}\right)$ & $\left(\mathcal{S}, \mathcal{S}_{2}\right)$ & $\left(\mathcal{S}, \mathcal{S}_{3}\right)$ & $\left(\mathcal{S}, \mathcal{S}_{4}\right)$ & $\left(\mathcal{S}, \mathcal{S}_{5}\right)$ & Ranking \\
\hline $\operatorname{Jac}_{w}\left(\mathcal{S}, \mathcal{S}_{r}\right)$ & 0.3697 & 0.4635 & 0.285 & 0.6618 & 0.3711 & $\mathcal{S}_{4} \geq \mathcal{S}_{2} \geq \mathcal{S}_{5} \geq \mathcal{S}_{1} \geq \mathcal{S}_{3}$ \\
$\operatorname{Dic}_{w}\left(\mathcal{S}, \mathcal{S}_{r}\right)$ & 0.5131 & 0.6042 & 0.4382 & 0.7468 & 0.5116 & $\mathcal{S}_{4} \geq \mathcal{S}_{2} \geq \mathcal{S}_{5} \geq \mathcal{S}_{1} \geq \mathcal{S}_{3}$ \\
$\operatorname{Cos}_{w}\left(\mathcal{S}, \mathcal{S}_{\mathbf{r}}\right)$ & 0.5239 & 0.6342 & 0.4561 & 0.7199 & 0.5173 & $\mathcal{S}_{4} \geq \mathcal{S}_{2} \geq \mathcal{S}_{1} \geq \mathcal{S}_{2} \geq \mathcal{S}_{3}$ \\
\hline
\end{tabular}

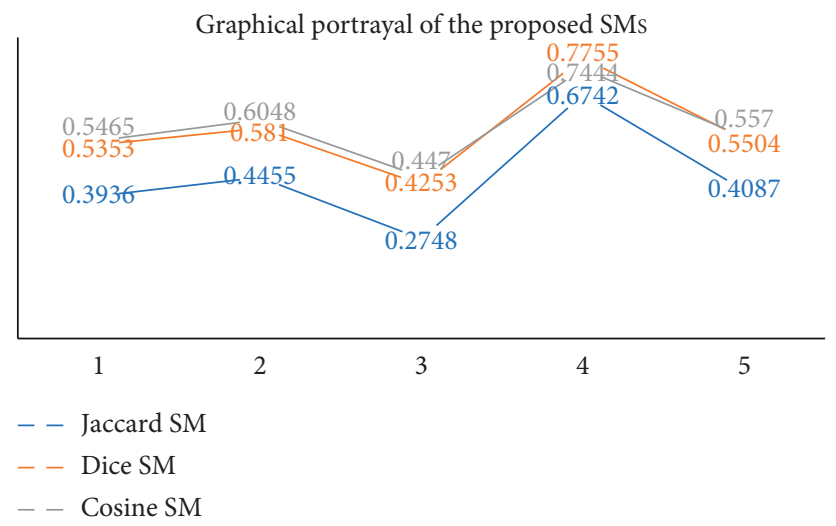

FIGURE 1: The graphical depiction of the deciphered VSMs between $\mathcal{S}$ and $\mathcal{S}_{r}(r=1,2,3,4,5)$.

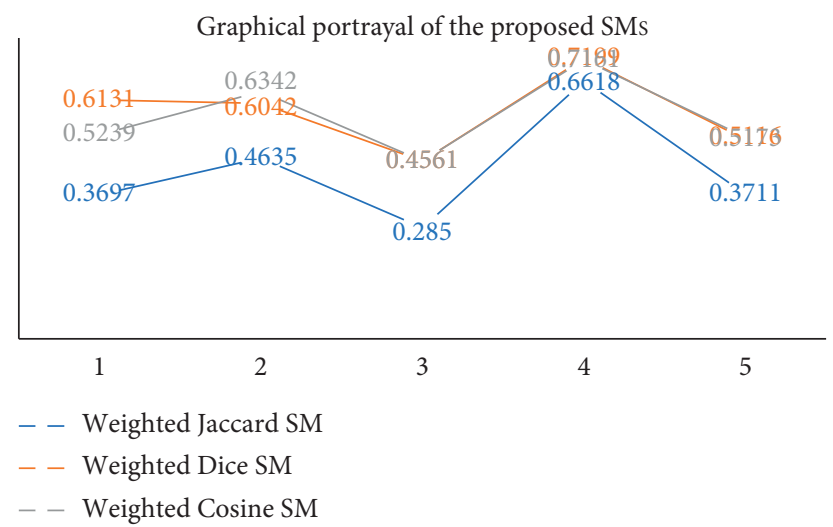

Figure 2: The graphical depiction of the deciphered WVSMs between $\mathcal{S}$ and $\mathcal{S}_{r}(r=1,2,3,4,5)$. 


$$
\begin{aligned}
\mathcal{S}_{4}= & \left\{\left(x_{1},\left\{\left\{0.4 e^{i 2 \pi(0.6)}, 0.6 e^{i 2 \pi(0.7)}\right\},\left\{0.15 e^{i 2 \pi(0.1)}, 0.3 e^{i 2 \pi(0.2)}\right\}\right\}\right),\left(x_{2},\left\{\left\{0.3 e^{i 2 \pi(0.4)}\right\},\left\{0.6 e^{i 2 \pi(0.5)}\right\}\right\}\right),\right. \\
& \left(x_{3},\left\{\left\{0.4 e^{i 2 \pi(0.3)}, 0.2 e^{i 2 \pi(0.4)}, 0.6 e^{i 2 \pi(0.5)}\right\},\left\{0.2 e^{i 2 \pi(0.4)}, 0.15 e^{i 2 \pi(0.3)}\right\}\right\}\right),\left(x_{4},\left\{\left\{0.5 e^{i 2 \pi(0.7)}, 0.3 e^{i 2 \pi(0.6)}\right\},\left\{0.4 e^{i 2 \pi(0.2)}\right\}\right\}\right) \\
& \left.\left(x_{5},\left\{\left\{0.6 e^{i 2 \pi(0.4)}, 0.55 e^{i 2 \pi(0.55)}, 0.3 e^{i 2 \pi(0.35)}\right\},\left\{0.35 e^{i 2 \pi(0.25)}, 0.15 e^{i 2 \pi(0.3)}, 0.1 e^{i 2 \pi(0.2)}\right\}\right\}\right)\right\} \\
\mathcal{S}_{5}= & \left\{\left(x_{1},\left\{\left\{0.3 e^{i 2 \pi(0.2)}, 0.2 e^{i 2 \pi(0.45)}\right\},\left\{0.5 e^{i 2 \pi(0.1)}, 0.4 e^{i 2 \pi(0.3)}\right\}\right\}\right),\left(x_{2},\left\{\left\{0.45 e^{i 2 \pi(0.6)}, 0.7 e^{i 2 \pi(0.55)}\right\},\left\{0.25 e^{i 2 \pi(0.35)}\right\}\right\}\right),\right. \\
& \left(x_{3},\left\{\left\{0.2 e^{i 2 \pi(0.35)}\right\},\left\{0.6 e^{i 2 \pi(0.6)}\right\}\right\}\right),\left(x_{4},\left\{\left\{0.7 e^{i 2 \pi(0.5)}\right\},\left\{0.15 e^{i 2 \pi(0.2)}, 0.25 e^{i 2 \pi(0.3)}\right\}\right\}\right) \\
& \left.\left(x_{5},\left\{\left\{0.4 e^{i 2 \pi(0.3)}, 0.25 e^{i 2 \pi(0.45)}, 0.1 e^{i 2 \pi(0.5)}\right\},\left\{0.5 e^{i 2 \pi(0.4)}, 0.5 e^{i 2 \pi(0.3)}, 0.3 e^{i 2 \pi(0.35)}\right\}\right\}\right)\right\} .
\end{aligned}
$$

Now an unknown building substance which needs to be identified is as follows:

$$
\begin{aligned}
\mathcal{S}= & \left\{\left(x_{1},\left\{\left\{\left\{0.7 e^{i 2 \pi(0.4)}, 0.3 e^{i 2 \pi(0.6)}\right\},\left\{0.2 e^{i 2 \pi(0.2)}, 0.1 e^{i 2 \pi(0.3)}\right\}\right\}\right\}\right),\left(x_{2},\left\{\left\{0.7 e^{i 2 \pi(0.6)}\right\},\left\{0.2 e^{i 2 \pi(0.3)}\right\}\right\}\right)\right. \\
& \left(x_{3},\left\{\left\{0.6 e^{i 2 \pi(0.5)}, 0.5 e^{i 2 \pi(0.4)}, 0.4 e^{i 2 \pi(0.7)}\right\},\left\{0.3 e^{i 2 \pi(0.1)}, 0.1 e^{i 2 \pi(0.2)}\right\}\right\}\right),\left(x_{4},\left\{\left\{0.4 e^{i 2 \pi(0.5)}, 0.6 e^{i 2 \pi(0.8)}\right\},\left\{0.15 e^{i 2 \pi(0.1)}\right\}\right\}\right) \\
& \left.\left(x_{5},\left\{\left\{0.5 e^{i 2 \pi(0.6)}, 0.4 e^{i 2 \pi(0.5)}, 0.2 e^{i 2 \pi(0.4)}\right\},\left\{0.3 e^{i 2 \pi(0.2)}, 0.1 e^{i 2 \pi(0.2)}, 0.2 e^{i 2 \pi(0.3)}\right\}\right\}\right)\right\} .
\end{aligned}
$$

The goal of this reliance is to sort the unknown building substance $\mathcal{S}$ in one of the arrangements $\mathcal{S}_{r}(r=1,2,3,4,5)$. For this, the deciphered VSMs (Jaccard, Dice, and Cosine) are used to check the similarity from $\delta$ to $\mathcal{S}_{r}(r=1,2,3,4,5)$ and calculations are given in Tables 1 and 2 .

As indicated by the above-figured estimations provided in Table 1, we basically notice that the similarity level between $\mathcal{S}$ and $\mathcal{S}_{4}$ is the best one as implantation by all VSMs. This characterizes that VSMs (Jaccard, Dice, and Cosine) give out the unknown building substance $\mathcal{S}$ to the known building substance $\mathcal{S}_{4}$ reliant on the standard of the best degree of similarity. Situating of the researched VSMs between and $\mathcal{S}_{r}(r=1,2,3,4,5)$ is likewise given in Table 1 . The graphical depiction of the deciphered VSMs between $\mathcal{S}$ and $\mathcal{S}_{r}(r=1,2,3,4,5)$ is exhibited in Figure 1 .

The weight of segments has exceptional importance to consider in certifiable powerful issues. If we surmise the greatness of segments $x_{k}(k=1,2,3,4,5)$ be $w_{k}(0.2,0.25$, $0.3,0.15,0.1)$, respectively. Now the interpreted WVSMs are used to choose the similarity from $\delta$ and $\mathcal{S}_{r}(r=1,2,3,4,5)$ and estimations are given in Table 2.

As indicated by the above-figured estimations provided in Table 2, we basically notice that the similarity level between $\mathcal{S}$ and $\mathcal{S}_{4}$ is the best one as implantation by all
WVSMs. This characterizes that WVSMs give out the unknown building substance $\mathcal{S}$ to the known building substance $\mathcal{S}_{4}$ reliant on the standard of the best degree of similarity. Situating of the researched VSMs between $\mathcal{S}$ and $\mathcal{S}_{r}(r=1,2,3,4,5)$ is likewise given in Table 2 . The graphical depiction of the deciphered VSMs between $\mathcal{S}$ and $\mathcal{S}_{r}(r=$ $1,2,3,4,5)$ is exhibited in Figure 2.

5.2. Medical Diagnosis. In this section, an altered algorithm for medical diagnosis is planned, which is improved depending on Xiao's algorithm [38]. The new algorithm uses the complex dual hesitant fuzzy similarity and distance measures that acquire glorious leads to application.

Example 4. Particular diseases have different signs. The medical diagnosis trust in the victim's signs to assess what kind of disease the victim has. The victim's signs are a set of signs and unknown diseases are a set of diagnostic diseases. A set of diagnoses is given by $\mathbb{D}=\left\{\mathbb{D}_{1}\right.$ (Diabetes), $\mathbb{D}_{2}$ (Malaria), $\mathbb{D}_{3}$ (Heart problem), $\mathbb{D}_{4}($ Flu $), \mathbb{D}_{5}$ (Hepatitis A) $\}$ and a set of signs is given by $X=\left\{x_{1}\right.$ (Fatigue), $x_{2}$ (Fever), $x_{3}$ (Heartpain), $x_{4}$ (Cough), $x_{5}$ (Abdominal pain) $\}$. The victim's signs can be given in the structure of CDHFSs as follows: 
TABLE 3: Estimations of the interpreted VSMs.

\begin{tabular}{|c|c|c|c|c|c|c|}
\hline Similarity measures & $\left(\mathbb{D}, \mathbb{D}_{1}\right)$ & $\left(\mathbb{D}, \mathbb{D}_{2}\right)$ & $\left(\mathbb{D}, \mathbb{D}_{3}\right)$ & $\left(\mathbb{D}, \mathbb{D}_{4}\right)$ & $\left(\mathbb{D}, \mathbb{D}_{5}\right)$ & Ranking \\
\hline $\operatorname{Jac}\left(\mathbb{P}, \mathbb{D}_{r}\right)$ & 0.1975 & 0.749 & 0.3781 & 0.3133 & 0.7702 & $\mathbb{D}_{2} \geq \mathbb{D}_{5} \geq \mathbb{D}_{3} \geq \mathbb{D}_{4} \geq \mathbb{D}_{1}$ \\
\hline $\operatorname{Dic}\left(\mathbb{P}, \mathbb{D}_{r}\right)$ & 0.3154 & 0.7977 & 0.4825 & 0.5178 & 0.6759 & $\mathbb{D}_{2} \geq \mathbb{D}_{5} \geq \mathbb{D}_{4} \geq \mathbb{D}_{3} \geq \mathbb{D}_{1}$ \\
\hline $\operatorname{Cos}\left(\mathbb{P}, \mathbb{D}_{r}\right)$ & 0.3328 & 0.8189 & 0.4865 & 0.4854 & 0.7159 & $\mathbb{D}_{2} \geq \mathbb{D}_{5} \geq \mathbb{D}_{3} \geq \mathbb{D}_{4} \geq \mathbb{D}_{1}$ \\
\hline
\end{tabular}

TABLE 4: Estimations of the interpreted WVSMs.

\begin{tabular}{lcccccc}
\hline Similarity measures & $\left(\mathbb{D}, \mathbb{D}_{1}\right)$ & $\left(\mathbb{D}, \mathbb{D}_{2}\right)$ & $\left(\mathbb{D}, \mathbb{D}_{3}\right)$ & $\left(\mathbb{D}, \mathbb{D}_{4}\right)$ & $\left(\mathbb{D}, \mathbb{D}_{5}\right)$ & Ranking \\
\hline $\operatorname{Jac}_{w}\left(\mathbb{P}, \mathbb{D}_{r}\right)$ & 0.2154 & 0.9221 & 0.3307 & 0.3136 & 0.7831 & $\mathbb{D}_{2} \geq \mathbb{D}_{5} \geq \mathbb{D}_{3} \geq \mathbb{D}_{4} \geq \mathbb{D}_{1}$ \\
$\operatorname{Dic}_{w}\left(\mathbb{P}, \mathbb{D}_{r}\right)$ & 0.3369 & 0.8173 & 0.4358 & 0.5009 & 0.7423 & $\mathbb{D}_{2} \geq \mathbb{D}_{5} \geq \mathbb{D}_{4} \geq \mathbb{D}_{3} \geq \mathbb{D}_{1}$ \\
$\operatorname{Cos}_{w}\left(\mathbb{P}, \mathbb{D}_{r}\right)$ & 0.3488 & 0.8407 & 0.4405 & 0.4829 & 0.7794 & $\mathbb{D}_{2} \geq \mathbb{D}_{5} \geq \mathbb{D}_{4} \geq \mathbb{D}_{3} \geq \mathbb{D}_{1}$ \\
\hline
\end{tabular}

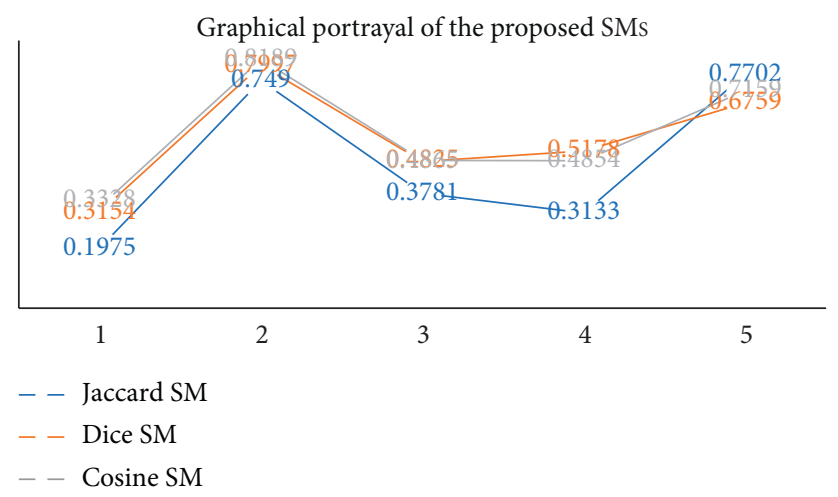

Figure 3: The graphical depiction of the deciphered VSMs between $\mathbb{P}$ and $\mathbb{D}_{r}(r=1,2,3,4,5)$.

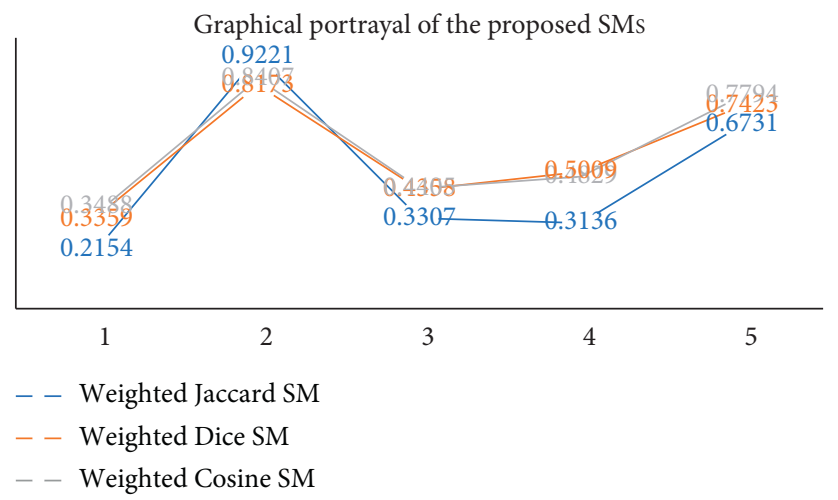

Figure 4: The graphical depiction of the deciphered WVSMs between $\mathbb{P}$ and $\mathbb{D}_{r}(r=1,2,3,4,5)$.

$$
\begin{aligned}
\mathbb{P}(\text { Victim })= & \left\{\left(x_{1},\left\{\left\{0.3 e^{i 2 \pi(0.2)}\right\}\left\{0.65 e^{i 2 \pi(0.7)}, 0.5 e^{i 2 \pi(0.6)}\right\}\right\}\right),\left(x_{2},\left\{\left\{0.8 e^{i 2 \pi(0.6)}, 0.55 e^{i 2 \pi(0.55)}, 0.45 e^{i 2 \pi(0.8)}\right\},\right.\right.\right. \\
& \left.\left.\left\{0.1 e^{i 2 \pi(0.15)}, 0.15 e^{i 2 \pi(0.1)}, 0.1 e^{i 2 \pi(0.05)}\right\}\right\}\right),\left(x_{3},\left\{\left\{0.45 e^{i 2 \pi(0.55)}\right\},\left\{0.25 e^{i 2 \pi(0.35)}\right\}\right\}\right) \\
& \left.\left(x_{4},\left\{\left\{0.35 e^{i 2 \pi(0.65)}, 0.65 e^{i 2 \pi(0.5)}\right\},\left\{0.15 e^{i 2 \pi(0.25)}, 0.3 e^{i 2 \pi(0.3)}\right\}\right\}\right),\left(x_{5},\left\{\left\{0.5 e^{i 2 \pi(0.3)}, 0.3 e^{i 2 \pi(0.6)}\right\},\left\{0.2 e^{i 2 \pi(0.25)}\right\}\right\}\right)\right\} .
\end{aligned}
$$


The signs of each disease $\mathbb{D}_{r}(r=1,2,3,4,5)$ can be represented in CDHFSs as follows:

$$
\begin{aligned}
& \mathbb{D}_{1} \text { (Diabetes) }=\left\{\left(x_{1},\left\{\left\{0.65 e^{i 2 \pi(0.7)}, 0.5 e^{i 2 \pi(0.6)}, 0.4 e^{i 2 \pi(0.5)}\right\},\left\{0.15 e^{i 2 \pi(0.2)}, 0.25 e^{i 2 \pi(0.15)}, 0.3 e^{i 2 \pi(0.25)}\right\}\right\}\right),\right. \\
& \left(x_{2},\left\{\left\{0.05 e^{i 2 \pi(0.15)}\right\},\left\{0.55 e^{i 2 \pi(0.8)}\right\}\right\}\right),\left(x_{3},\left\{\left\{0.1 e^{i 2 \pi(0.01)}\right\},\left\{0.15 e^{i 2 \pi(0.65)}\right\}\right\}\right), \\
& \left.\left(x_{4},\left\{\left\{0.5 e^{i 2 \pi(0.1)}\right\},\left\{0.5 e^{i 2 \pi(0.3)}, 0.1 e^{i 2 \pi(0.2)}\right\}\right\}\right),\left(x_{5},\left\{\left\{0.01 e^{i 2 \pi(0.05)}\right\},\left\{0.05 e^{i 2 \pi(0.34)}, 0.15 e^{i 2 \pi(0.25)}\right\}\right\}\right)\right\}, \\
& \mathbb{D}_{2} \text { (Malaria) }=\left\{\left(x_{1},\left\{\left\{0.3 e^{i 2 \pi(0.4)}\right\}\left\{0.45 e^{i 2 \pi(0.5)}, 0.6 e^{i 2 \pi(0.45)}\right\}\right\}\right),\left(x_{2},\left\{\left\{0.7 e^{i 2 \pi(0.55)}, 0.65 e^{i 2 \pi(0.6)}, 0.7 e^{i 2 \pi(0.5)}\right\},\right.\right.\right. \\
& \left.\left.\left\{0.25 e^{i 2 \pi(0.1)}, 0.2 e^{i 2 \pi(0.15)}, 0.25 e^{i 2 \pi(0.35)}\right\}\right\}\right),\left(x_{3},\left\{\left\{0.5 e^{i 2 \pi(0.4)}\right\},\left\{0.3 e^{i 2 \pi(0.4)}\right\}\right\}\right) \\
& \left(x_{4},\left\{\left\{0.75 e^{i 2 \pi(0.45)}, 0.55 e^{i 2 \pi(0.6)}\right\},\left\{0.2 e^{i 2 \pi(0.25)}, 0.15 e^{i 2 \pi(0.3)}\right\}\right\}\right) \text {, } \\
& \left.\left(x_{5},\left\{\left\{0.7 e^{i 2 \pi(0.4)}, 0.2 e^{i 2 \pi(0.3)}\right\},\left\{0.25 e^{i 2 \pi(0.5)}\right\}\right\}\right)\right\}, \\
& \mathbb{D}_{3}(\text { Heart problem })=\left\{\left(x_{1},\left\{\left\{0.45 e^{i 2 \pi(0.3)}\right\},\left\{0.35 e^{i 2 \pi(0.5)}\right\}\right\}\right),\left(x_{2},\left\{\left\{0.05 e^{i 2 \pi(0.15)}\right\},\left\{0.55 e^{i 2 \pi(0.2)}\right\}\right\}\right)\right. \\
& \left(x_{3},\left\{\left\{0.6 e^{i 2 \pi(0.5)}, 0.8 e^{i 2 \pi(0.6)}, 0.7 e^{i 2 \pi(0.4)}\right\},\left\{0.1 e^{i 2 \pi(0.35)}, 0.15 e^{i 2 \pi(0.25)}\right\}\right\}\right), \\
& \left(x_{4},\left\{\left\{0.4 e^{i 2 \pi(0.5)}, 0.7 e^{i 2 \pi(0.6)}\right\},\left\{0.2 e^{i 2 \pi(0.1)}, 0.25 e^{i 2 \pi(0.35)}\right\}\right\}\right), \\
& \left.\left(x_{5},\left\{\left\{0.35 e^{i 2 \pi(0.1)}\right\},\left\{0.5 e^{i 2 \pi(0.35)}, 0.3 e^{i 2 \pi(0.65)}\right\}\right\}\right)\right\}, \\
& \mathbb{D}_{4}(\mathrm{Flu})=\left\{\left(x_{1},\left\{\left\{0.4 e^{i 2 \pi(0.3)}\right\},\left\{0.2 e^{i 2 \pi(0.35)}\right\}\right\}\right),\left(x_{2},\left\{\left\{0.5 e^{i 2 \pi(0.7)}, 0.4 e^{i 2 \pi(0.6)}\right\},\left\{0.4 e^{i 2 \pi(0.2)}, 0.3 e^{i 2 \pi(0.15)}\right\}\right\}\right),\right. \\
& \left(x_{3},\left\{\left\{0.1 e^{i 2 \pi(0.05)}\right\},\left\{0.35 e^{i 2 \pi(0.5)}, 0.5 e^{i 2 \pi(0.3)}\right\}\right\}\right),\left(x_{4},\left\{\left\{0.6 e^{i 2 \pi(0.3)}, 0.55 e^{i 2 \pi(0.45)}, 0.5 e^{i 2 \pi(0.55)}\right\},\right.\right. \\
& \left.\left.\left.\left\{0.2 e^{i 2 \pi(0.35)}, 0.3 e^{i 2 \pi(0.25)}, 0.15 e^{i 2 \pi(0.2)}\right\}\right\}\right),\left(x_{5},\left\{\left\{0.25 e^{i 2 \pi(0.4)}, 0.5 e^{i 2 \pi(0.36)}\right\},\left\{0.2 e^{i 2 \pi(0.3)}\right\}\right\}\right)\right\} \\
& \mathbb{D}_{5}(\text { Hepatitis A })=\left\{\left(x_{1},\left\{\left\{0.2 e^{i 2 \pi(0.45)}\right\},\left\{0.7 e^{i 2 \pi(0.35)}, 0.6 e^{i 2 \pi(0.3)}\right\}\right\}\right),\left(x_{2},\left\{\left\{0.5 e^{i 2 \pi(0.05)}\right\},\left\{0.4 e^{i 2 \pi(0.45)}\right\}\right\}\right),\right. \\
& \left(x_{3},\left\{\left\{0.25 e^{i 2 \pi(0.5)}\right\},\left\{0.6 e^{i 2 \pi(0.25)}\right\}\right\}\right),\left(x_{4},\left\{\left\{0.2 e^{i 2 \pi(0.35)}, 0.3 e^{i 2 \pi(0.6)}\right\},\left\{0.2 e^{i 2 \pi(0.2)}\right\}\right\}\right), \\
& \left.\left(x_{5},\left\{\left\{0.8 e^{i 2 \pi(0.5)}, 0.7 e^{i 2 \pi(0.7)}, 0.5 e^{i 2 \pi(0.55)}\right\},\left\{0.15 e^{i 2 \pi(0.25)}, 0.1 e^{i 2 \pi(0.2)}, 0.05 e^{i 2 \pi(0.25)}\right\}\right\}\right)\right\} .
\end{aligned}
$$

The goal of this reliance is to sort the disease of the victim $\mathbb{P}$ in one of the diseases $\mathbb{D}_{r}(r=1,2,3,4,5)$. For this, the deciphered VSMs (Jaccard, Dice, and Cosine) are used to check the similarity from $\mathbb{P}$ to $\mathbb{D}_{r}(r=1,2,3,4,5)$ and calculations are given in Tables 3 and 4 .

As indicated by the above-figured estimations provided in Table 3, we basically notice that the similarity level between $\mathbb{P}$ and $\mathbb{D}_{2}$ is the best one as implantation by all VSMs. This characterizes that VSMs (Jaccard, Dice, and Cosine) give out that the victim has malaria reliant on the standard of the best degree of similarity. Situating of the researched VSMs between $\mathbb{P}$ and $\mathbb{D}_{r}(r=1,2,3,4,5)$ is likewise given in Table 3 . The graphical depiction of the deciphered VSMs between $\mathbb{P}$ and $\mathbb{D}_{r}(r=1,2,3,4,5)$ is exhibited in Figure 3.
The weight of segments has exceptional importance to consider in certifiable powerful issues. If we surmise the greatness of segments $x_{k}(k=1,2,3,4,5)$ be $w_{k}(0.2,0.25,0.3,0.15,0.1)$, respectively. Now the interpreted WVSMs are used to choose the similarity from $\mathbb{P}$ and $\mathbb{D}_{r}(r=$ $1,2,3,4,5)$ and estimations are given in Table 4.

As indicated by the above-figured estimations provided in Table 4, we basically notice that the similarity level between $\mathbb{P}$ and $\mathbb{D}_{2}$ is the best one as implantation by all WVSMs. This characterizes that WVSMs give out that the victim has malaria reliant on the standard of the best degree of similarity. Situating of the researched WVSMs between $\mathbb{P}$ and $\mathbb{D}_{r}(r=1,2,3,4,5)$ is likewise given in Table 4 . The graphical depiction of the deciphered VSMs between $\mathbb{P}$ and $\mathbb{D}_{r}(r=1,2,3,4,5)$ is exhibited in Figure 4 . 
TABLE 5: Comparison among interpreted and some existing SMs for Example 5.

\begin{tabular}{|c|c|c|}
\hline Method & Score value & Ranking \\
\hline $\begin{array}{l}\text { Wang et al. } \\
{[33]}\end{array}$ & $\begin{array}{c}s^{1}\left(\mathcal{S}, \mathcal{S}_{1}\right)=0.8273, s^{1}\left(\mathcal{S}, \mathcal{S}_{2}\right)=0.914, s^{1}\left(\mathcal{S}, \mathcal{S}_{3}\right)=0.8277, s^{1}\left(\mathcal{S}, \mathcal{S}_{4}\right)=0.8945 \\
s^{1}\left(\mathcal{S}, \mathcal{S}_{5}\right)=0.797\end{array}$ & $\mathcal{S}_{2} \geq \mathcal{S}_{4} \geq \mathcal{S}_{3} \geq \mathcal{S}_{1} \geq \mathcal{S}_{5}$ \\
\hline $\begin{array}{l}\text { Wang et al. } \\
{[33]}\end{array}$ & $s^{2}\left(\mathcal{S}, \mathcal{S}_{1}\right)=0.69, s^{2}\left(\mathcal{S}, \mathcal{S}_{2}\right)=0.691, s^{2}\left(\mathcal{S}, \mathcal{S}_{3}\right)=0.68, s^{2}\left(\mathcal{S}, \mathcal{S}_{4}\right)=0.70, s^{2}\left(\mathcal{S}, \mathcal{S}_{5}\right)=0.63$ & $\mathcal{S}_{4} \geq \mathcal{S}_{2} \geq \mathcal{S}_{1} \geq \mathcal{S}_{3} \geq \mathcal{S}_{5}$ \\
\hline $\begin{array}{l}\text { Wang et al. } \\
{[33]}\end{array}$ & $\begin{array}{c}s^{3}\left(\mathcal{S}, \mathcal{S}_{1}\right)=0.7525, s^{3}\left(\mathcal{S}, \mathcal{S}_{2}\right)=0.7216, s^{3}\left(\mathcal{S}, \mathcal{S}_{3}\right)=0.6958, s^{3}\left(\mathcal{S}, \mathcal{S}_{4}\right)=0.7425 \\
s^{3}\left(\mathcal{S}, \mathcal{S}_{5}\right)=0.6725\end{array}$ & $\mathcal{S}_{1} \geq \mathcal{S}_{4} \geq \mathcal{S}_{2} \geq \mathcal{S}_{3} \geq \mathcal{S}_{5}$ \\
\hline Explored SM & $\begin{array}{c}\operatorname{Jac}\left(\mathcal{S}, \mathcal{S}_{1}\right)=0.4148, \operatorname{Jac}\left(\mathcal{S}, \mathcal{S}_{2}\right)=0.4765, \operatorname{Jac}\left(\mathcal{S}, \mathcal{S}_{3}\right)=0.2716, \operatorname{Jac}\left(\mathcal{S}, \mathcal{S}_{4}\right)=0.587 \\
\operatorname{Jac}\left(\mathcal{S}, \mathcal{S}_{5}\right)=0.3582\end{array}$ & $\mathcal{S}_{4} \geq \mathcal{S}_{2} \geq \mathcal{S}_{1} \geq \mathcal{S}_{5} \geq \mathcal{S}_{3}$ \\
\hline Explored SM & $\begin{array}{c}\operatorname{Dic}\left(\mathcal{S}, \mathcal{S}_{1}\right)=0.5624, \operatorname{Dic}\left(\mathcal{S}, \mathcal{S}_{2}\right)=0.6103, \operatorname{Dic}\left(\mathcal{S}, \mathcal{S}_{3}\right)=0.426, \operatorname{Dic}\left(\mathcal{S}, \mathcal{S}_{4}\right)=0.7291 \\
\operatorname{Dic}\left(\mathcal{S}, \mathcal{S}_{5}\right)=0.5123\end{array}$ & $\mathcal{S}_{4} \geq \mathcal{S}_{2} \geq \mathcal{S}_{1} \geq \mathcal{S}_{5} \geq \mathcal{S}_{3}$ \\
\hline Explored SM & $\begin{array}{c}\operatorname{Cos}\left(\mathcal{S}, \mathcal{S}_{1}\right)=0.5696, \operatorname{Cos}\left(\mathcal{S}, \mathcal{S}_{2}\right)=0.6322, \operatorname{Cos}\left(\mathcal{S}, \mathcal{S}_{3}\right)=0.4539, \operatorname{Cos}\left(\mathcal{S}, \mathcal{S}_{4}\right)=0.7062 \\
\operatorname{Cos}\left(\mathcal{S}, \mathcal{S}_{5}\right)=0.5248\end{array}$ & $\mathcal{S}_{4} \geq \mathcal{S}_{2} \geq \mathcal{S}_{1} \geq \mathcal{S}_{5} \geq \mathcal{S}_{3}$ \\
\hline
\end{tabular}

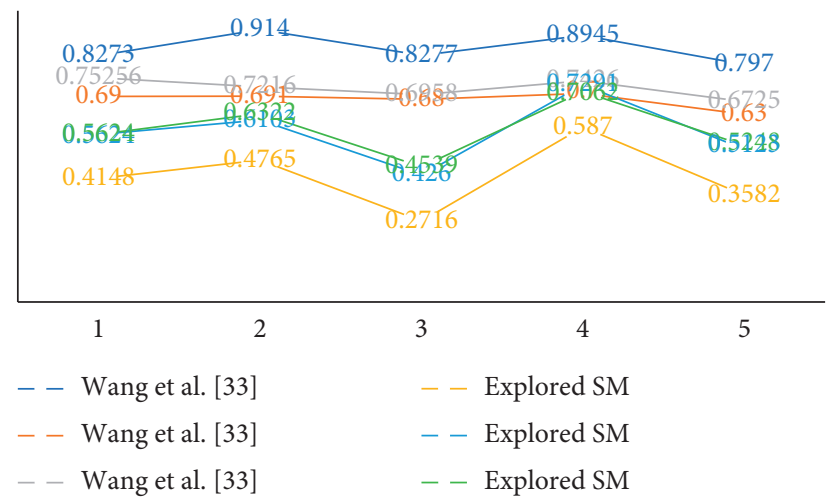

FIgURE 5: The graphical depiction between the proposed and existing SMs for Example 5.

TABLE 6: Comparison among interpreted and some existing SMs for Example 3.

\begin{tabular}{lcc}
\hline Method & Score value & Ranking \\
\hline Wang et al. [33] & Failed & Failed \\
Wang et al. [33] & Failed & Failed \\
Wang et al. [33] & Failed & Failed \\
Explored SM & $\operatorname{Jac}\left(\mathcal{S}, \mathcal{S}_{1}\right)=0.3936, \operatorname{Jac}\left(\mathcal{S}, \mathcal{S}_{2}\right)=0.4455, \operatorname{Jac}\left(\mathcal{S}, \mathcal{S}_{3}\right)=0.2748, \operatorname{Jac}\left(\mathcal{S}, \mathcal{S}_{4}\right)=0.6742$, & $\mathcal{S}_{4} \geq \mathcal{S}_{2} \geq \mathcal{S}_{5} \geq \mathcal{S}_{1} \geq \mathcal{S}_{3}$ \\
& $\operatorname{Jac}\left(\mathcal{S}, \mathcal{S}_{5}\right)=0.4087$ & $\mathcal{S}_{4} \geq \mathcal{S}_{2} \geq \mathcal{S}_{5} \geq \mathcal{S}_{1} \geq \mathcal{S}_{3}$ \\
Explored SM & $\operatorname{Dic}\left(\mathcal{S}, \mathcal{S}_{1}\right)=0.5353, \operatorname{Dic}\left(\mathcal{S}, \mathcal{S}_{2}\right)=0.581, \operatorname{Dic}\left(\mathcal{S}, \mathcal{S}_{3}\right)=0.4253, \operatorname{Dic}\left(\mathcal{S}, \mathcal{S}_{4}\right)=0.7755$, & $\operatorname{Dic}\left(\mathcal{S}, \mathcal{S}_{5}\right)=0.5504$, \\
Explored SM & $\operatorname{Cos}\left(\mathcal{S}, \mathcal{S}_{1}\right)=0.5465, \operatorname{Cos}\left(\mathcal{S}, \mathcal{S}_{1}\right)=0.6048, \operatorname{Cos}\left(\mathcal{S}, \mathcal{S}_{3}\right)=0.447, \operatorname{Cos}\left(\mathcal{S}, \mathcal{S}_{4}\right)=0.7444$, & $\mathcal{S}_{4} \geq \mathcal{S}_{2} \geq \mathcal{S}_{5} \geq \mathcal{S}_{1} \geq \mathcal{S}_{3}$ \\
\hline
\end{tabular}

\section{Comparison}

In this section, we showed the feasibility and focal points of the deciphered SMs by differentiating some existing SMs.

Example 5. Its feed that amounts from developments by an organization is legitimately corresponding to the standard of building substances they use. An appropriate review of the building substance before development is the confirmation of good building measures. The building substances to be utilized ought to be carefully checked before applying. The best possible check and equalization arrangement of investigation approve the manufacturers to utilize the correct substances for developments to improve the standard of their task. Five known building substances $\mathcal{S}_{r}(r=1,2,3,4,5)$ which are given in the DHFSs structure are as follows: 


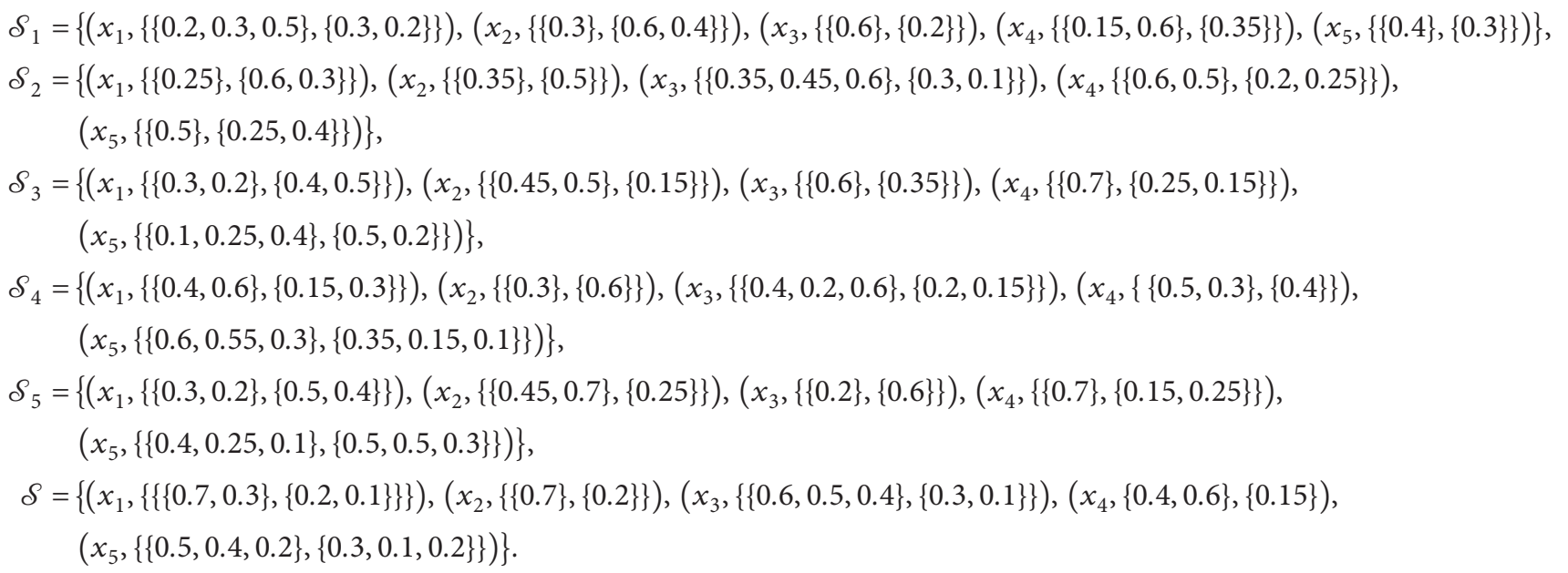

We convert the above information which is given in the form of DHFSs into CDHFSs by putting $1=e^{0}$ as presented below:

$$
\begin{aligned}
& \mathcal{S}_{1}=\left\{\left(x_{1},\left\{\left\{0.2 e^{i 2 \pi(0.0)}, 0.3 e^{i 2 \pi(0.0)}, 0.5 e^{i 2 \pi(0.0)}\right\},\left\{0.3 e^{i 2 \pi(0.0)}, 0.2 e^{i 2 \pi(0.0)}\right\}\right\}\right),\left(x_{2},\left\{\left\{0.3 e^{i 2 \pi(0.0)}\right\},\left\{0.6 e^{i 2 \pi(0.0)}, 0.4 e^{i 2 \pi(0.0)}\right\}\right\}\right)\right. \\
& \left.\left(x_{3},\left\{\left\{0.6 e^{i 2 \pi(0.0)}\right\},\left\{0.2 e^{i 2 \pi(0.0)}\right\}\right\}\right),\left(x_{4},\left\{\left\{0.15 e^{i 2 \pi(0.0)}, 0.6 e^{i 2 \pi(0.0)}\right\},\left\{0.35 e^{i 2 \pi(0.0)}\right\}\right\}\right),\left(x_{5},\left\{\left\{0.4 e^{i 2 \pi(0.0)}\right\},\left\{0.3 e^{i 2 \pi(0.0)}\right\}\right\}\right)\right\}, \\
& \mathcal{S}_{2}=\left\{\left(x_{1},\left\{\left\{0.25 e^{i 2 \pi(0.0)}\right\},\left\{0.6 e^{i 2 \pi(0.0)}, 0.3 e^{i 2 \pi(0.0)}\right\}\right\}\right),\left(x_{2},\left\{\left\{0.35 e^{i 2 \pi(0.0)}\right\},\left\{0.5 e^{i 2 \pi(0.0)}\right\}\right\}\right),\right. \\
& \left(x_{3},\left\{\left\{0.35 e^{i 2 \pi(0.0)}, 0.45 e^{i 2 \pi(0.0)}, 0.6 e^{i 2 \pi(0.0)}\right\},\left\{0.3 e^{i 2 \pi(0.0)}, 0.1 e^{i 2 \pi(0.0)}\right\}\right\}\right),\left(x_{4},\left\{\left\{0.6 e^{i 2 \pi(0.0)}, 0.5 e^{i 2 \pi(0.0)}\right\},\right.\right. \\
& \left.\left.\left.\left\{0.2 e^{i 2 \pi(0.0)}, 0.25 e^{i 2 \pi(0.0)}\right\}\right\}\right),\left(x_{5},\left\{\left\{0.5 e^{i 2 \pi(0.0)}\right\},\left\{0.25 e^{i 2 \pi(0.0)}, 0.4 e^{i 2 \pi(0.0)}\right\}\right\}\right)\right\}, \\
& \mathcal{S}_{3}=\left\{\left(x_{1},\left\{\left\{0.3 e^{i 2 \pi(0.0)}, 0.2 e^{i 2 \pi(0.0)}\right\},\left\{0.4 e^{i 2 \pi(0.0)}, 0.5 e^{i 2 \pi(0.0)}\right\}\right\}\right),\left(x_{2},\left\{\left\{0.45 e^{i 2 \pi(0.0)}, 0.5 e^{i 2 \pi(0.0)}\right\},\left\{0.15 e^{i 2 \pi(0.0)}\right\}\right\}\right),\right. \\
& \left(x_{3},\left\{\left\{0.6 e^{i 2 \pi(0.0)}\right\},\left\{0.35 e^{i 2 \pi(0.0)}\right\}\right\}\right),\left(x_{4},\left\{\left\{0.7 e^{i 2 \pi(0.0)}\right\},\left\{0.25 e^{i 2 \pi(0.0)}, 0.15 e^{i 2 \pi(0.0)}\right\}\right\}\right), \\
& \left.\left(x_{5},\left\{\left\{0.1 e^{i 2 \pi(0.0)}, 0.25 e^{i 2 \pi(0.0)}, 0.4 e^{i 2 \pi(0.0)}\right\},\left\{0.5 e^{i 2 \pi(0.0)}, 0.2 e^{i 2 \pi(0.0)}\right\}\right\}\right)\right\} \\
& \mathcal{S}_{4}=\left\{\left(x_{1},\left\{\left\{0.4 e^{i 2 \pi(0.0)}, 0.6 e^{i 2 \pi(0.0)}\right\},\{0.15,0.3\}\right\}\right),\left(x_{2},\left\{\left\{0.3 e^{i 2 \pi(0.0)}\right\},\left\{0.6 e^{i 2 \pi(0.0)}\right\}\right\}\right),\right. \\
& \left(x_{3},\left\{\left\{0.4 e^{i 2 \pi(0.0)}, 0.2 e^{i 2 \pi(0.0)}, 0.6 e^{i 2 \pi(0.0)}\right\},\left\{0.2 e^{i 2 \pi(0.0)}, 0.15 e^{i 2 \pi(0.0)}\right\}\right\}\right),\left(x_{4},\left\{\left\{0.5 e^{i 2 \pi(0.0)}, 0.3 e^{i 2 \pi(0.0)}\right\},\left\{0.4 e^{i 2 \pi(0.0)}\right\}\right\}\right), \\
& \left.\left(x_{5},\left\{\left\{0.6 e^{i 2 \pi(0.0)}, 0.55 e^{i 2 \pi(0.0)}, 0.3 e^{i 2 \pi(0.0)}\right\},\left\{0.35 e^{i 2 \pi(0.0)}, 0.15 e^{i 2 \pi(0.0)}, 0.1 e^{i 2 \pi(0.0)}\right\}\right\}\right)\right\}, \\
& \mathcal{S}_{5}=\left\{\left(x_{1},\left\{\left\{0.3 e^{i 2 \pi(0.0)}, 0.2 e^{i 2 \pi(0.0)}\right\},\left\{0.5 e^{i 2 \pi(0.0)}, 0.4 e^{i 2 \pi(0.0)}\right\}\right\}\right),\left(x_{2},\left\{\left\{0.45 e^{i 2 \pi(0.0)}, 0.7 e^{i 2 \pi(0.0)}\right\},\left\{0.25 e^{i 2 \pi(0.0)}\right\}\right\}\right),\right. \\
& \left(x_{3},\left\{\left\{0.2 e^{i 2 \pi(0.0)}\right\},\left\{0.6 e^{i 2 \pi(0.0)}\right\}\right\}\right),\left(x_{4},\left\{\left\{0.7 e^{i 2 \pi(0.0)}\right\},\left\{0.15 e^{i 2 \pi(0.0)}, 0.25 e^{i 2 \pi(0.0)}\right\}\right\}\right) \\
& \left.\left(x_{5},\left\{\left\{0.4 e^{i 2 \pi(0.0)}, 0.25 e^{i 2 \pi(0.0)}, 0.1 e^{i 2 \pi(0.0)}\right\},\left\{0.5 e^{i 2 \pi(0.0)}, 0.5 e^{i 2 \pi(0.0)}, 0.3 e^{i 2 \pi(0.0)}\right\}\right\}\right)\right\}, \\
& \mathcal{S}=\left\{\left(x_{1},\left\{\left\{\left\{0.7 e^{i 2 \pi(0.0)}, 0.3 e^{i 2 \pi(0.0)}\right\},\left\{0.2 e^{i 2 \pi(0.0)}, 0.1 e^{i 2 \pi(0.0)}\right\}\right\}\right\}\right),\left(x_{2},\left\{\left\{0.7 e^{i 2 \pi(0.0)}\right\},\left\{0.2 e^{i 2 \pi(0.0)}\right\}\right\}\right),\right. \\
& \left(x_{3},\left\{\{0.6,0.5,0.4\},\left\{0.3 e^{i 2 \pi(0.0)}, 0.1 e^{i 2 \pi(0.0)}\right\}\right\}\right),\left(x_{4},\left\{0.4 e^{i 2 \pi(0.0)}, 0.6 e^{i 2 \pi(0.0)}\right\},\left\{0.15 e^{i 2 \pi(0.0)}\right\}\right), \\
& \left.\left(x_{5},\left\{\left\{0.5 e^{i 2 \pi(0.0)}, 0.4 e^{i 2 \pi(0.0)}, 0.2 e^{i 2 \pi(0.0)}\right\},\left\{0.3 e^{i 2 \pi(0.0)}, 0.1 e^{i 2 \pi(0.0)}, 0.2 e^{i 2 \pi(0.0)}\right\}\right\}\right)\right\} .
\end{aligned}
$$

For instance (25), we have to find that the obscure building substance $\mathcal{S}$ has a spot with the foreordained building material $\mathcal{S}_{r}(r=1,2,3,4,5)$. The data of model (25) introduced looking like DHFSs. By some current SMs for DHFSs, we identified the closeness among $\mathcal{S}$ and $\mathcal{S}_{r}(r=$ $1,2,3,4,5)$ as shown in Table 5 . As we have $1=e^{0}$, then the 


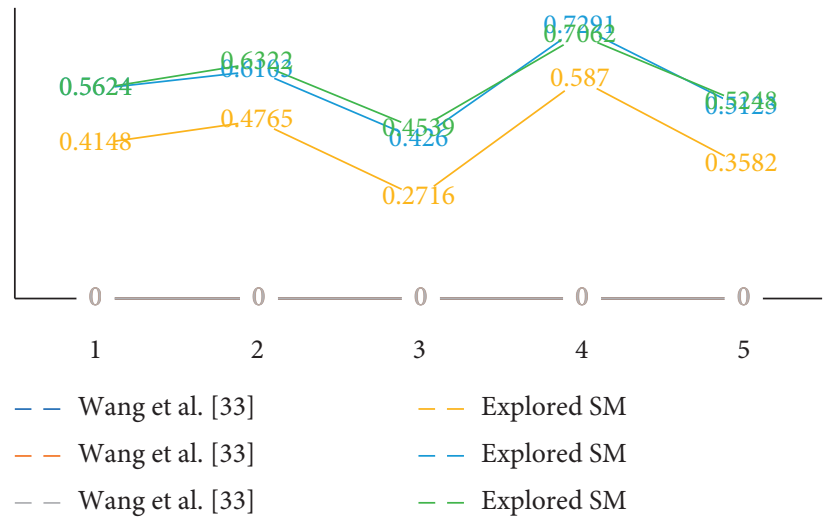

FIGURE 6: The graphical depiction between the proposed and existing SMs for Example 3.

data, given in model (25), were transformed into CDHFSs. Now through deciphered SMs, we identified the closeness among $\mathcal{S}$ and $r(r=1,2,3,4,5)$ in like manner as shown in Table 5. Our deciphered SMs (Jaccard, Dice, and Cosine) exhibited that obscure building substance $\delta$ has a spot with predefined building substance $\mathcal{S}_{4}$ in the light of the fact that the similitude among $\mathcal{S}$ and $\mathcal{S}_{4}$ is most conspicuous one. The positioning of the explored and existing SMs is similarly presented in Table 5. The graphical depiction of the correlation of the proposed and existing SMs is shown in Figure 5.

By and by we analyze the correlation among deciphered and existing SMs for instance (17). For instance (17), the data are given in the structure of CDHFSs. We understand that no SM exists in the composition to comprehend such data. The current SMs are insufficient to find the closeness among $\mathcal{S}$ and $\mathcal{S}_{r}(r=1,2,3,4,5)$ as shown in Table 6. From Table 6, we see that the data introduced in model (17) are just plausible by the investigated SMs. The deciphered SMs find the similitude among $\mathcal{S}$ and $\mathcal{S}_{r}(r=1,2,3,4,5)$ as shown in Table 6. Our deciphered SMs (Jaccard, Dice, and Cosine) exhibited that dubious building material $\delta$ has a spot with the foreordained building material $\mathcal{S}_{4}$ in light of the face that the closeness among $\mathcal{S}$ and $\mathcal{S}_{4}$ is most imperative one. The situating of the interpreted SMs is furthermore presented in Table 6. The graphical depiction of the assessment of proposed and existing SMs is shown in Figure 6.

In this correlation, we stood out our researched SMs from some current SMs described by Wang et al. [33]. From the above discussion, indisputably, our proposed SMs can talk extra fluffy data and put it extensively in conditions, all things considered, issues. In the view of CDHFS, we investigated the SMs, our SMs are dynamically pleasing for authentic issues than the current SMs, and our SMs are more expansive than the current SMs.

\section{Conclusion}

The notion of CDHFS is a mixture of two alterations called CFS and DHFS. CDHFS creates two degrees called truth valued and falsely valued as a subset of a unit disc in a complex plane and is a capable method to adapt to questionable and unusual data, in real-life decisions. In this paper, we investigated the notion of CDHFS and its operational laws. The novel approach of CIvDHFS and its fundamental laws are also investigated and furthermore advocated with the assistance of models. Further, the VSMs, WVSMs, hybrid vector similarity measures, and weighted hybrid vector similarity measures are also explored. These SMs are applied in the environment of pattern recognition and medical diagnosis to assess the proficiency and feasibility of the interpreted measures. We additionally unraveled some numerical examples utilizing the setup measures. Moreover, we examined the dependability and legitimacy of the proposed SMs by comparing them with some existing SMs. In this paper, the point of interest, relative examination, and graphical portrayal of the investigated SMs and existing SMs are also discussed in detail.

In future, we will work on linear Diophantine fuzzy sets (LDFS) [16, 45, 46], complex fuzzy sets [47], complex q-rung orthopair fuzzy sets [48-52], etc. [53-59].

\section{Data Availability}

The data used in this article are artificial and hypothetical, and anyone can use these data before prior permission by just citing this article.

\section{Conflicts of Interest}

The authors declare that they have no conflicts of interest.

\section{Acknowledgments}

This paper was supported by "Algebra and Applications Research Unit, Faculty of Science, Prince of Songkla University."

\section{References}

[1] L. A. Zadeh, "Fuzzy sets," Information and Control, vol. 8, no. 3, pp. 338-353, 1965.

[2] L. A. Zadeh, "The concept of a linguistic variable and its application to approximate reasoning-I," Information Sciences, vol. 8, no. 3, pp. 199-249, 1975.

[3] L. A. Zadeh, "The concept of a linguistic variable and its application to approximate reasoning-II," Information Sciences, vol. 8, no. 4, pp. 301-357, 1975. 
[4] L. A. Zadeh, "The concept of a linguistic variable and its application to approximate reasoning-III," Information Sciences, vol. 9, no. 1, pp. 43-80, 1975.

[5] K. T. Atanassov, "Intuitionistic fuzzy sets," in Intuitionistic Fuzzy Sets, pp. 1-137, Springer, Berlin, Germany, 1999.

[6] K. T. Atanassov, "Interval valued intuitionistic fuzzy sets," in Intuitionistic Fuzzy Sets, pp. 139-177, Springer, Berlin, Germany, 1999.

[7] Z. Xu, "A method based on linguistic aggregation operators for group decision making with linguistic preference relations," Information Sciences, vol. 166, no. 1-4, pp. 19-30, 2004.

[8] D. J. Dubois, Fuzzy Sets and Systems: Theory and Applications, Vol. 144, Academic Press, Cambridge, MA, USA, 1980.

[9] R. R. Yager, "On the theory of bags," International Journal of General Systems, vol. 13, no. 1, pp. 23-37, 1986.

[10] T. Baležentis and S. Zeng, "Group multi-criteria decision making based upon interval-valued fuzzy numbers: an extension of the MULTIMOORA method," Expert Systems with Applications, vol. 40, no. 2, pp. 543-550, 2013.

[11] W. Su, Y. Yang, C. Zhang, and S. Zeng, "Intuitionistic fuzzy decision-making with similarity measures and OWA operator," International Journal of Uncertainty, Fuzziness and Knowledge-Based Systems, vol. 21, no. 2, pp. 245-262, 2013.

[12] B. Peng, C. Ye, and S. Zeng, "Some intuitionist fuzzy weighted geometric distance measures and their application to group decision making," International Journal of Uncertainty, Fuzziness and Knowledge-Based Systems, vol. 22, no. 5, pp. 699-715, 2014.

[13] C. Zhang, C. Chen, D. Streimikiene, and T. Balezentis, "Intuitionistic fuzzy MULTIMOORA approach for multicriteria assessment of the energy storage technologies," Applied Soft Computing, vol. 79, pp. 410-423, 2019.

[14] J. Zhou, T. Baležentis, and D. Streimikiene, "Normalized weighted Bonferroni harmonic mean-based intuitionistic fuzzy operators and their application to the sustainable selection of search and rescue robots," Symmetry, vol. 11, no. 2, p. $218,2019$.

[15] S. Zeng, S.-M. Chen, and K.-Y. Fan, "Interval-valued intuitionistic fuzzy multiple attribute decision making based on nonlinear programming methodology and TOPSIS method," Information Sciences, vol. 506, pp. 424-442, 2020.

[16] M. Riaz and M. R. Hashmi, "Linear diophantine fuzzy set and its applications towards multi-attribute decision-making problems," Journal of Intelligent \& Fuzzy Systems, vol. 37, no. 4, pp. 5417-5439, 2019.

[17] V. Torra, "Hesitant fuzzy sets," International Journal of Intelligent Systems, vol. 25, pp. 529-539, 2010.

[18] Z. Xu and M. Xia, "On distance and correlation measures of hesitant fuzzy information," International Journal of Intelligent Systems, vol. 26, no. 5, pp. 410-425, 2011.

[19] D. Li, W. Zeng, and J. Li, "New distance and similarity measures on hesitant fuzzy sets and their applications in multiple criteria decision making," Engineering Applications of Artificial Intelligence, vol. 40, pp. 11-16, 2015.

[20] S. Zeng and Y. Xiao, "A method based on TOPSIS and distance measures for hesitant fuzzy multiple attribute decision making," Technological and Economic Development of Economy, vol. 24, no. 3, pp. 969-983, 2018.

[21] J. Hu, Y. Yang, X. Zhang, and X. Chen, "Similarity and entropy measures for hesitant fuzzy sets," International Transactions in Operational Research, vol. 25, no. 3, pp. 857-886, 2018.
[22] Z. Xu and M. Xia, "Distance and similarity measures for hesitant fuzzy sets," Information Sciences, vol. 181, no. 11, pp. 2128-2138, 2011.

[23] W. Zeng, D. Li, and Q. Yin, "Distance and similarity measures between hesitant fuzzy sets and their application in pattern recognition," Pattern Recognition Letters, vol. 84, pp. 267-271, 2016.

[24] Z. Mu, S. Zeng, and T. Baležentis, "A novel aggregation principle for hesitant fuzzy elements," Knowledge-Based Systems, vol. 84, pp. 134-143, 2015.

[25] J. C. R. Alcantud and V. Torra, "Decomposition theorems and extension principles for hesitant fuzzy sets," Information Fusion, vol. 41, pp. 48-56, 2018.

[26] H. Liao and Z. Xu, "Subtraction and division operations over hesitant fuzzy sets," Journal of Intelligent \& Fuzzy Systems, vol. 27, no. 1, pp. 65-72, 2014.

[27] K. Bisht and S. Kumar, "Fuzzy time series forecasting method based on hesitant fuzzy sets," Expert Systems with Applications, vol. 64, pp. 557-568, 2016.

[28] J. C. R. Alcantud and A. Giarlotta, "Necessary and possible hesitant fuzzy sets: a novel model for group decision making," Information Fusion, vol. 46, pp. 63-76, 2019.

[29] X. Zhang and Z. Xu, "Novel distance and similarity measures on hesitant fuzzy sets with applications to clustering analysis," Journal of Intelligent \& Fuzzy Systems, vol. 28, no. 5, pp. 2279-2296, 2015.

[30] B. Farhadinia and E. Herrera-Viedma, "Multiple criteria group decision making method based on extended hesitant fuzzy sets with unknown weight information," Applied Soft Computing, vol. 78, pp. 310-323, 2019.

[31] N. Chen, Z. Xu, and M. Xia, "Interval-valued hesitant preference relations and their applications to group decision making," Knowledge-Based Systems, vol. 37, pp. 528-540, 2013.

[32] B. Zhu, Z. Xu, and M. Xia, "Dual hesitant fuzzy sets," Journal of Applied Mathematics, vol. 2012, Article ID 879629, 13 pages, 2012.

[33] L. Wang, Q. Wang, S. Xu, and M. Ni, "Distance and similarity measures of dual hesitant fuzzy sets with their applications to multiple attribute decision making," in Proceedings of the 2014 IEEE International Conference on Progress in Informatics and Computing, Shanghai, China, May 2014.

[34] B. Zhu and Z. Xu, "Some results for dual hesitant fuzzy sets," Journal of Intelligent \& Fuzzy Systems, vol. 26, no. 4, pp. 1657-1668, 2014.

[35] S. K. Tyagi, "Correlation coefficient of dual hesitant fuzzy sets and its applications," Applied Mathematical Modelling, vol. 39, no. 22, pp. 7082-7092, 2015.

[36] B. Farhadinia, "Correlation for dual hesitant fuzzy sets and dual interval-valued hesitant fuzzy sets," International Journal of Intelligent Systems, vol. 29, no. 2, pp. 184-205, 2014.

[37] D. Ramot, R. Milo, M. Friedman, and A. Kandel, "Complex fuzzy sets," IEEE Transactions on Fuzzy Systems, vol. 10, no. 2, pp. 171-186, 2002.

[38] F. Xiao and W. Ding, "Divergence measure of Pythagorean fuzzy sets and its application in medical diagnosis," Applied Soft Computing, vol. 79, pp. 254-267, 2019.

[39] M. Munir, H. Kalsoom, K. Ullah, T. Mahmood, and Y.-M. Chu, “T-spherical fuzzy Einstein hybrid aggregation operators and their applications in multi-attribute decision making problems," Symmetry, vol. 12, no. 3, p. 365, 2020.

[40] K. Ullah, T. Mahmood, and H. Garg, "Evaluation of the performance of search and rescue robots using $\mathrm{T}$-spherical 
fuzzy hamacher aggregation operators," International Journal of Fuzzy Systems, vol. 22, no. 2, pp. 570-582, 2020.

[41] T. Mahmood, K. Ullah, Q. Khan, and N. Jan, "An approach toward decision-making and medical diagnosis problems using the concept of spherical fuzzy sets," Neural Computing and Applications, vol. 31, no. 11, pp. 7041-7053, 2019.

[42] Z. Ali and T. Mahmood, "Complex neutrosophic generalised dice similarity measures and their application to decision making," CAAI Transactions on Intelligence Technology, vol. 5, no. 2, pp. 78-87, 2020.

[43] Z. Ali and T. Mahmood, "Maclaurin symmetric mean operators and their applications in the environment of complex q-rung orthopair fuzzy sets," Computational and Applied Mathematics, vol. 39, p. 161, 2020.

[44] P. Liu, T. Mahmood, and Z. Ali, "Complex Q-rung orthopair fuzzy aggregation operators and their applications in multiattribute group decision making," Information, vol. 11, no. 1, p. $5,2020$.

[45] M. Riaz, K. Naeem, and D. Afzal, "Pythagorean m-polar fuzzy soft sets with TOPSIS method for MCGDM," Punjab University Journal of Mathematics, vol. 52, no. 3, pp. 21-46, 2020.

[46] M. Riaz and M. R. Hashmi, "Soft rough Pythagorean m-polar fuzzy sets and Pythagorean m-polar fuzzy soft rough sets with application to decision-making," Computational and Applied Mathematics, vol. 39, no. 1, p. 16, 2020.

[47] P. Liu, Z. Ali, and T. Mahmood, "The distance measures and cross-entropy based on complex fuzzy sets and their application in decision making," Journal of Intelligent \& Fuzzy Systems, vol. 39, no. 3, pp. 3351-3374, 2020.

[48] P. Liu, Z. Ali, and T. Mahmood, "A method to multi-attribute group decision-making problem with complex q-rung orthopair linguistic information based on Heronian mean operators," International Journal of Computational Intelligence Systems, vol. 12, no. 2, pp. 1465-1496, 2019.

[49] H. Garg, J. Gwak, T. Mahmood, and Z. Ali, "Power aggregation operators and VIKOR methods for complex q-rung orthopair fuzzy sets and their applications," Mathematics, vol. 8, no. 4, p. 538, 2020.

[50] P. Liu, Z. Ali, T. Mahmood, and N. Hassan, "Group decisionmaking using complex q-rung orthopair fuzzy Bonferroni mean," International Journal of Computational Intelligence Systems, vol. 13, no. 1, pp. 822-851, 2020.

[51] T. Mahmood and Z. Ali, "Entropy measure and TOPSIS method based on correlation coefficient using complex q-rung orthopair fuzzy information and its application to multi-attribute decision making," Soft Computing, pp. 1-27, 2020.

[52] H. Garg, Z. Ali, and T. Mahmood, "Algorithms for complex interval-valued q-rung orthopair fuzzy sets in decision making based on aggregation operators, AHP, and TOPSIS," Expert Systems, Article ID e12609, 2020.

[53] Z. Ali, T. Mahmood, and M.-S. Yang, "Complex T-spherical fuzzy aggregation operators with application to multi-attribute decision making," Symmetry, vol. 12, no. 8, p. 1311, 2020.

[54] T. Mahmood, U. Ur Rehman, Z. Ali, and T. Mahmood, "Hybrid vector similarity measures based on complex hesitant fuzzy sets and their applications to pattern recognition and medical diagnosis," Journal of Intelligent \& Fuzzy Systems, pp. 1-22, 2020.

[55] M. A. Ramadan, H. S. Osheba, and A. R. Hadhoud, "A highly efficient and accurate finite iterative method for solving linear two-dimensional Fredholm fuzzy integral equations of the second kind using triangular functions," Mathematical Problems in Engineering, vol. 2020, Article ID 2028763, 16 pages, 2020.
[56] J. Hamaydi and N. Qatanani, "Computational methods for solving linear fuzzy Volterra integral equation," Journal of Applied Mathematics, vol. 2017, Article ID 2417195, 12 pages, 2017.

[57] R. M. Shabestari, R. Ezzati, and T. Allahviranloo, "Solving fuzzy Volterra integrodifferential equations of fractional order by Bernoulli wavelet method," Advances in Fuzzy Systems, vol. 2018, Article ID 5603560, 11 pages, 2018.

[58] S. Zeng, X. Peng, T. Baležentis, and D. Streimikiene, "Prioritization of low-carbon suppliers based on Pythagorean fuzzy group decision making with self-confidence level," Economic Research-Ekonomska Istraživanja, vol. 32, no. 1, pp. 1073-1087, 2019.

[59] S. Zeng, D. Luo, C. Zhang, and X. Li, "A correlation-based TOPSIS method for multiple attribute decision making with single-valued neutrosophic information," International Journal of Information Technology \& Decision Making, vol. 19, no. 1, pp. 343-358, 2020. 